\title{
XII.
}

\section{Per la storia della Sofistica greca.}

\author{
Di
}

\begin{abstract}
Alessandro Chiappelli in Napoli.
\end{abstract}
\author{
(Secondo Articolo.)
}

La possibilità di una applicazione etica della idea della natura, preparata dalla religione popolare greca che aveva sempre rappresentata la connessione tra $\mathrm{i}$ fenomeni fisici e morali, e più da vicino del pensiero speculativo che aveva raccolta la varietà dei fenomeni nell' unità della natura, dipendeva però da alcuni aspetti di questa idea, suscettivi di applicazioni morali. La natura si presenta come eguale dovunque, e quindi la sua legge è comune a tutti i popoli. La storia di Erodoto è tutta animata del sentimento di questa legge. Inoltre essa si manifesta in un ordine, dove ogni elemento s'aggira nella sua sfera; e come Anassimandro aveva chiamata áôxía la separazione degli elementi dalla materia originaria ${ }^{1}$ ), così Eraclito aveva detto che il sole non può uscire dal suo giro poichè lo punirebbero le Erini, tutrici della universale giustizia $\left(\delta c^{\prime}\left(\gamma_{1}\right)^{2}\right)$ : e come non è giusto che esca dal suo giro il

1). Simplic. Phys. 24, 18. Pel nostro fine è indifferente se in questo passo

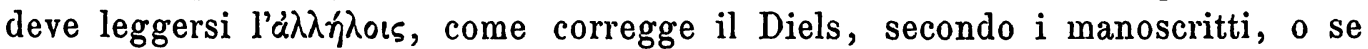
„l'ingiustizia“ si riferisce ai rapporti delle singole cose coll' ä $\pi \varepsilon \iota p o v . \quad$ cfr. Ziegler in Archiv f. Gesch. d. Phil. I, 1, 1887 p. 16-27.

2) Fr. 29. Byw. e 62. Ho rilevato già altrove (Atti della R. Accademia di scienze morale e politiche di Napoli. 1888 p. 140 ss.) come il concetto di Eraclito sia in antitesi evidente coll' altro di Anassimandro. Il concetto della $\Delta i x \eta$ è comune anche a Parmenìle v. $73 \mathrm{~s}$. (Stein.) 
sole, così non è lecito che il mare ecceda la sua naturale misura ${ }^{3}$ ). Un esempio di questo scambio di attributi etici e d'intuizioni fisiche all' età dei Sofisti, l'abbiamo nelle Fenicie d'Euripide, dove Giocasta invita Eteocle ad onorare l'eguaglianza (ioótrss) come quella che unisce gli uomini agli uomini, facendoli amici e alleati, e stabilisce fra loro la giustizia (vóucusv), mentre dà la misura (uśtpov) alle cose naturali, ordina il periodo dei giorni e delle notti, e la regolare vicenda degli anni ${ }^{4}$ ).

Un tal metodo di ragionare ci apparisce appunto nell' apologo di Heracles al bivio, contenuto nello scritto di Prodico che portava il titolo di ?:Spat. A chi lo guardi nel suo insieme (Xenoph. Memor. II, 1. 21-34) non può sfuggire che il conflitto morale fra

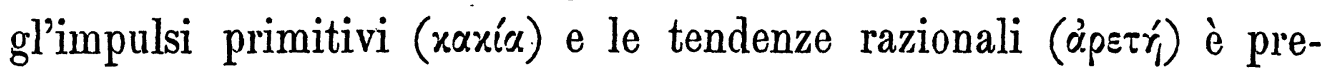
sentato come uno stato primitivo della coscienza, sul quale non ha esercitato ancora efficacia il complicato raffinamento della vita civile. Heracles è lindividuo nella semplicità della natura sua, e che ha quindi in sè il germe dell' opposte tendenze; ed ecco perchè è

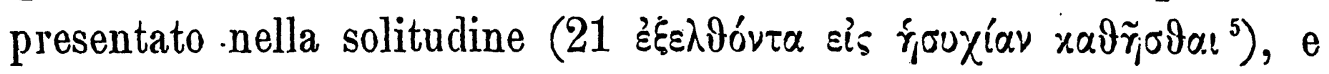
perchè Arete dichiara di fondare le sue speranze sulla natura di

3) Fr. 23. Byw. Si può supporre una allusione ironica a questa dottrina in Arisiofane Nub. 1290

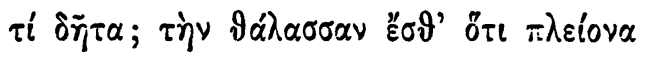

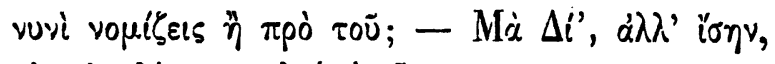

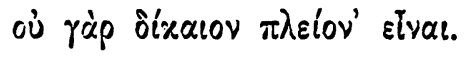

4) Phoeniss. 536-47

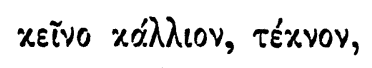

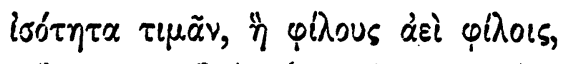

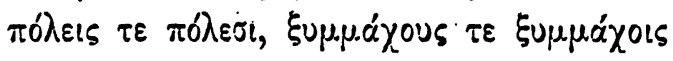

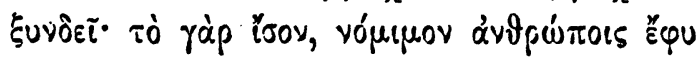

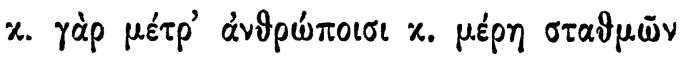

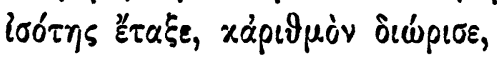

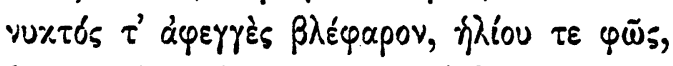

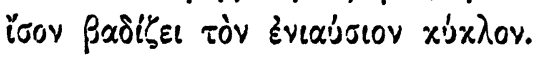

5) Che questa particolarità si trovasse nel racconto di Prodico si può rac-

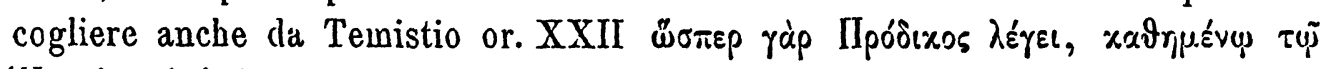

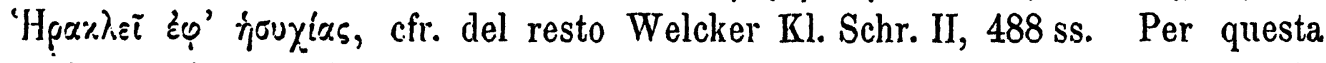
ragione mi pare che il Welcker s'apponga quando a p. 484 nega che nelle scene dei due $\lambda \alpha^{\prime}$ or d'Aristofane sia imitato il discorso di Prodico. Il punto di 


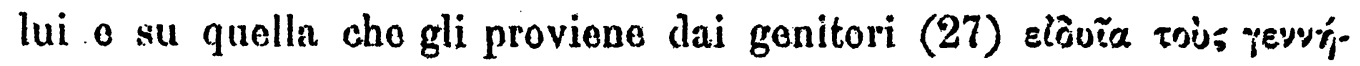

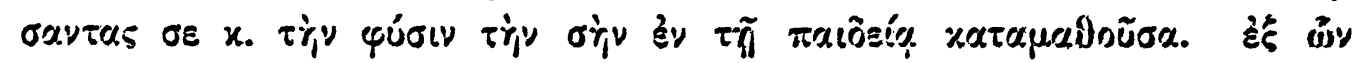
$\dot{\varepsilon} \lambda \pi(\zeta(1) x \tau \lambda$.$) .$

Per questo al fondo di questo insegnamento morale noi troviamo un Naturalismo eudemonistico che prepara l'etica naturalistica dei Cinici. Il prevalere della virtì ha la sua ragione in un dato naturale, cioc̀ in quel processo di compensazione onde il piacere è neutralizzato dal languore, dalla sazietà e dalla decadenza prematura; in altre parole in un motivo che nasce della applicazione d'un ragionamento fisico o d'un calcolo naturale all' ordine morale. Ed è appunto l'ordine divino della natura per cui il vizio è pena

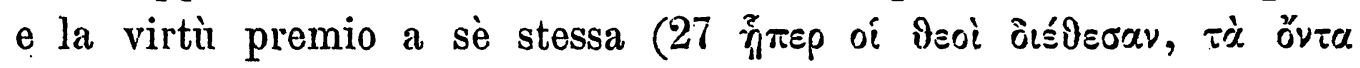
ôrrýropusl); e su questa considerazione si fonda il tono parenetico di tutto l'Apologo, che Senofonte contrappone ad Aristippo ${ }^{6}$ ). Spira da tutto l'insieme del mito un aura di semplicità e di candore come da una scena di natura, che lo rendeva, come pare, facilmente accessibile ad un pubblico numeroso (Ib. II 1, 21).

Che tale fosse l'intuizione dominante nell' insegnamento di Prodico, apparisce anche dal concetto che a lui viene attribuito

vista morale è tutto diverso, ma bisogna notare che si ricollega però in parte il Logos Dikaios al motivo stesso dell' apologo or Prodico, in quanto vi è proposto Heracles come esempio (Nubb. v. 1051). Confr. Massimo Tyr. Dissert. 20, 1 .

6) Questo spirito naturalistico dell' insegnamento di Prodico evidente nell' apologo di Heracles, ci è confërmato da uni notizia di Aristotele (Top. II, 6, 112 b, 22) e dello Scoliaste di Platone Schol. ad Pl. Phaedr. 267 B, (cfr. Xen. Mem. II, 1, 21; Plat. Protag. 337 b) dai quali sappiamo che Prodico aveva in-

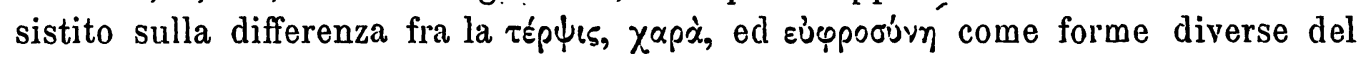
piacere. cfr. Heinze, Der Eudäm. in d. Gr. Phil. 1884 p. 724 ss. Berichte d. Sächs. Gesell. 1884 p. 326 ss. Così il detto di lui riferito da Platone (Euthyd. 308 e) accenne ad una specie d'avversione a coloro che stanno fia i filosofi e gli uomini politici. Questa stessa tendenza naturalistica nell' Etica si riproduce anche nelle scuole anti-edonistiche che si ricollegano a Prodico, come questa di Antistene. Noi sappiamo difatti che Antistene era familiare di Prodico (Xen. Symp. IV, 62), e anche se non dobbiamo credere che avesse trattato gli stessi argomenti in uno scritto su Heracles e Mida, come pare probabile dopo le ricerche del Susemihl, Jahrb. f. Philol. 1887 H. 3, 4 p. 207-14, sebbene lo sọstenga anche ora il Dümmler Akademika 1889 p. 156 ss. rimane sempre il fattọ chẹ Heracleș era l'eroe favorito dei Cinici (Diog. VI, 16. 18) 
nel Pseudoplatonico Erixia (397) ${ }^{7}$ ); cioè che della natura propria

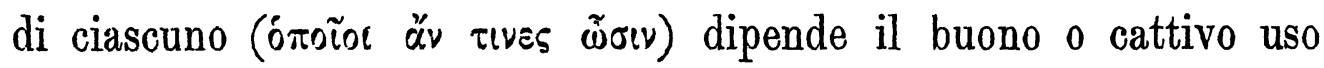
che gli uomini fanno delle cose, e che specialmente la ricchezza sia un bene solo per gli uomini saggi e virtuosi. E in questo stesso ordine di concetti si muoveva, per quanto si può argomentare dalle sparse notizie che ne'abbiamo, lo scritto di Prodico sui beni dell' agricoltura ${ }^{8}$ ). L'oggetto sembra ne fosse una rappresentazione d'una vita semplice, vicina alla natura primitiva, e un elogio della vita domestica ed agreste, la cui sostanza dovè esser poi riprodotta negli scritti dei Socratici, e sopratutto nell' Economico di Senofonte e in quello di Antistene (D. L. VI, 18). Sembra che Prodico vi ricollegasse il culto, i misteri religiosi e la benevolenza degli Dei verso gli uomini alla semplicità della vita campestre, della quale celebrava la bellezza e l'onestà, con quel senso quasi idillico delle origini e quella aspirazione all uno tempo antico, che trasparisce non solo dalle Baccanti di Euripide, che una tradizione antica fa discepolo di Prodico ${ }^{\circ}$ ), rappresentante il trionfo dei pri-

cfr. Welcker Kl. Schr. II, 483, che son quelli a cui allude Platone nel Filebo. Del resto noi sappiamo che i Cinici, che Platone e Aristotele chiamano di $\pi \alpha i-$ ócuto , negavano ogni valore alla educazione e alla cultura, e come più tardi gli Stoici, segnano una restaurazione del principio di natura.

7) La contradizione che vi è fra questo concetto e la dottrina espressa da Prodico poco dopo (398 c) dimostra solo la poca autorità che ha questo scritto come fonte storica. L'annoverarlo coll' Axioco alla letteratura cinica, come fa il Dümmler, Akademika p. 78, mi sembra per lo meno ardito.

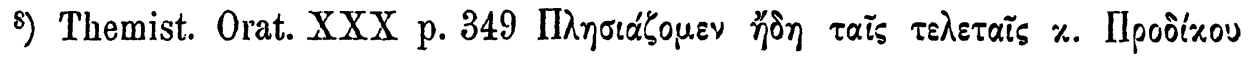

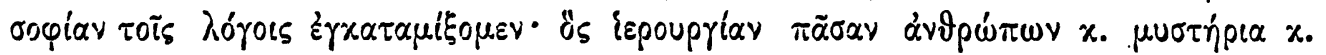

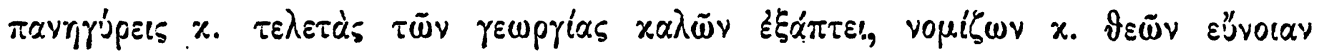

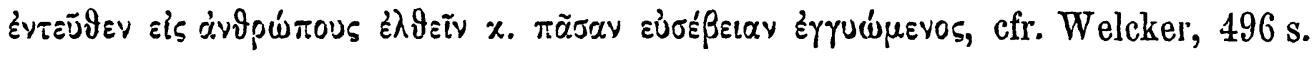
Strümpell, Gesch. d. theor. Phil. p. 14 s. Siebeck. Unters. z. Phil. d. Gr. 2 A. $188 \mathrm{~s}$ p. 37.

9) Gell. Noct. A. XV, 20, 4. Aristoph. Rạ. 1188. cf. Dümmler, Akademika, 61,257 .

E cosi intendiamo il modo onde Prodico spiegava l'origine dei culti e delle religioni che rivela in lui lo studio delle origini umane. Egli ne ricercava le origini nelle lontane oscurità del periodo preistorico. Gli uomini primitivi divinizzarono, secondo lui, tutto ciò che loro era utile; onde il pane era adorato come Demeter, il vino come Dionysos, il fuoco come Hefestos ecc. (Cic. nat Deor. I, 42. 118. Sext. Emp. Math. IX, 18; 15 s.) cfr. Welcker, op. c. 520 ss. A cui è da aggiungere Philodem. De Piet. Jr. c. 10. Doxographi 
mitivi culti dionisiaci, ma anche nel frammento 1 (Nauck), dove il poeta vaghoggia l'antica vita dell' Attica, l'età dei successori d'Erotteo. S'intende quindi come Prodico, secondo che apparisce dall' Axioco pseudoplatonico e forse anche, secondo la congettura del $W^{\text {elcker }}{ }^{10}$ ), dalla parodia d'Aristofane nella Parahasi degli Uccelli, in contrapposto a questa beatitudine delle origini, si rappresentasse come infelice la vita umana attuale.

Anche nella questione filologica sulla origine dei nomi, che aveva parte nell' insegnamento di Prodico (Cratyl. 384 b), sembra che questi sostenesse l'origine naturale non già dall' uso $\left.{ }^{1}\right)$ : La sinonimica derisa da Platone aveva fondamento nell' etimologia dei vocaboli, cioè la dottrina per cui ognuno di essi esprime naturalmente una cosa sola, e ciò per una somiglianza che corra fra il suono e la cosa espressa; sicchè si debbano distinguere accuratamente gli uni dagli altri. La ragione dei nomi era quindi per

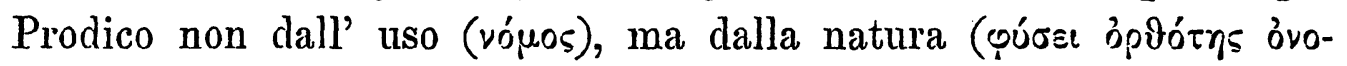
$\mu \alpha ́ \tau \omega \nu)$.

Ma una chiara e riflessa antitesi fra la natura e il vóuos, fra il diritto naturale e il diritto positivo, la troviamo rilevata per primo da Ippia, che ben può dirsi, per questo rispetto, l'Ugo Gro-. zio dell' antichità. Allo spirito greco non poteva sfuggire che il segno della natura anche nell' ordine morale è l'universalità e l'immutabilità. Quel pensiero che aveva fino a qui mirato a cogliere nella sparsa varietà dei fenomeni naturali l'unità sottostante, doveva esser disposto a tentare da prima di scoprire anche nella vita umana

Graec. 544 b. Ed Epiph. Haeres. III, 21. Doxogr. p. 591. Non mi pare quindi che questa spiegazione naturalistica delle religioni di Prodico debbe esser messa insieme colle negazioni religiose degli ultimi Sofisti come fanno lo Zeller op. cit. I, 1012 e il Köstlin op. cit. I, p. 226, bensi piuttosto si colleghi allo stesso principio di spiegazione che troviamo anche in Democrito e più chiaramente a una dottrina Orfica Abel, Orphica 1885 fr. 160-62 p. 216. cfr. O. Kern Archiv II, 3. p. 389. Dümmler, Akad., 162 ss.

10) cfr. all' incontro Dümmler, Akad. $157 \mathrm{~s}$.

11) Un resto di questi studi di Prodico sul linguaggio ci è conservato da Galeno De Pot. phys. 2,9, al qual frammento il Dümmler op. cit. p. 159 ricollega il Pseudoippocrate De Nat. hom. c. 5 (VI, 40 L.) e De Carn. c. 4 (VIII, 588 L.). 
il fondamento unico posto anche quivi della natura. D'altra parte i costumi, le tradizioni dei differenti popoli presentavano una infinita varietà. Le narrazioni dei logografi avevano richiamata l'attenzione sopra questi contrasti e in qualche modo avevano preparata la via alla Sofistica ${ }^{2}$ ); e sebbene la narrazione erodotea sia penetrata del sentimento "che vi è una legge morale superiore alle nazioni, pure Erodoto ripete il detto di Pindaro che „il Nomos è re", quando narra che a Dario i Greci rifutarono di mangiare le carni dei loro morti mentre gl'Indiani avevano sdegnato di arderle (Her. III, 38) ${ }^{13}$ ). Nè solo la varietà delle leggi e delle istituzioni presso i vari popoli, ma la variabilità loro nel moto accelerato delle nuove democrazie, e nella rivalità politica fra gli stati greci pareva stette a provare contro alla loro autorità riconoscinta fino a qui.

Tutti questi motivi suggerivano naturalmente l'idea che le istituzioni sociali sieno un prodotto artificiale e che in esse stia il germe delle discordie fra gli uomini. Ora Ippia, così erudito nella storia dei popoli, aveva trovato appunto e nelle condizioni del suo tempo e nella cultura propria il motivo della sua dottrina, ch'egli esprime nel Protagora ( $377 \mathrm{~b}$. s) „o cittadini qui.presenti, io penso che tutti siate affini, familiari e compaesani per natura, non per legge; poichè il simile è per natura affine col simile, ma la legge, tiranna degli uomini, in molte cose fa violenze contro la natura " ${ }^{14}$ ). E la ragione ne è questa che la politica anche per lui, come diremmo oggi, divide gli animi che la natura aveva uniti, che il diritto positivo non è che una degenerazione della natura. Egli vagheggia quindi la vita umana nelle sua condizione primitiva, cioè quale è data dalla coúosẹ, non come è formata dalla vita civile. Onde nell' Ippia Maggiore $282 \mathrm{~A}$ dice a Socrate $\varepsilon^{\prime} \omega \vartheta 0$.

12) Diels in Hermes XXII, 58 ss.

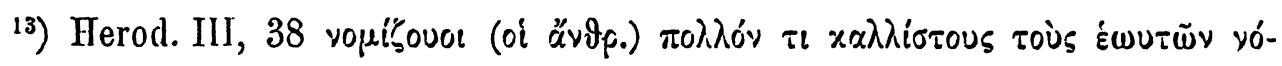
y.ovs ë\%so:ol eival.

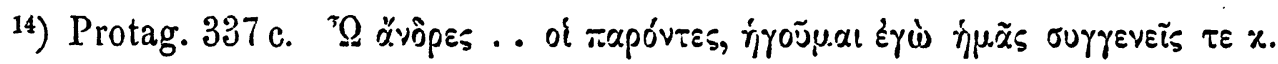

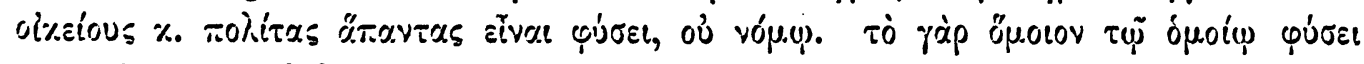

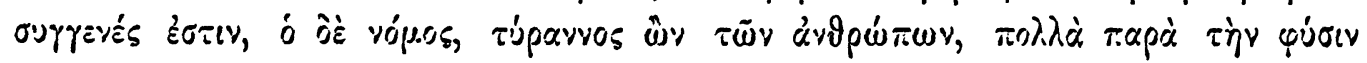

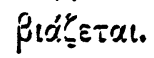




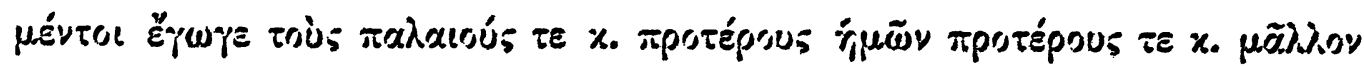

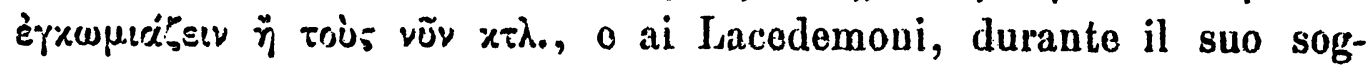
giorno in Sparta, afferma di aver narrato ( $285 \mathrm{~d}$ ) l'origine degli eroi, degli uomini, e della città, o cioè tutta la storia dell' anti-

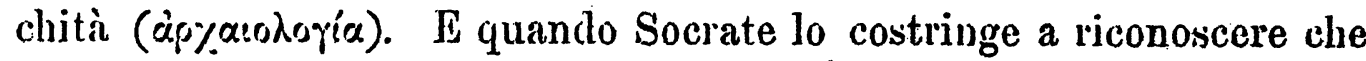
le leggi furono costituite per il bene degli stati ( $284 \mathrm{~d}$ ss.), e che le

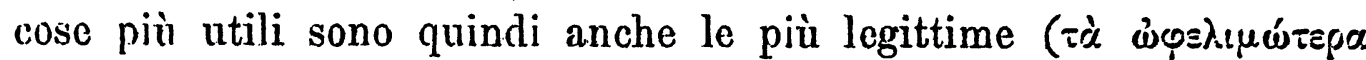

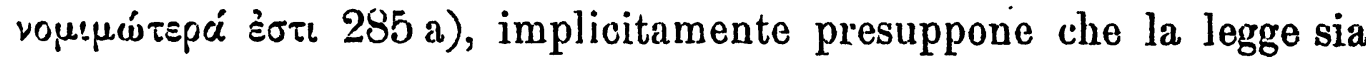
opera di convenzione ( 9 śoıs) riflessa ${ }^{15}$ ). Onde accetta bensi da Socrate la distinzione fra le leggi positive, e ciò che è legittimo o legge in sè stesso (vóucuov $\tau \varepsilon$ xai vóuos), ma soggiunge che sia propria di chi sa e frutto di ragione accorta ( $284 \mathrm{~d}$. e), mentre il volgo (oí $\pi 0 \lambda \lambda \lambda_{i}$ ) accetta il vóuos cosi come è costituito dei legislatori.

A scuoprire quindi la regola naturale del diritto egli paragonava le leggi di nazioni diverse, scegliendo ciò che hanno di comune, come fondamento di un sistema etico. Codesto aspetto del pensiero d'Ippia apparisce dal dialogo di lui col Socrate (Mem. IV. 4, 5-25), nel reciso contrasto fra la dottrina di Socrate che identifica il giusto col legale (vóuruov) e quella d'Ippia che non ammette una tale identità. Ambedue riconoscono nelle leggi positive il fondamento naturale, gli ärpaço vóure di Sofocle. Ma differiscono poi in ciò che mentre per Socrate la legge positiva comanda in quanto è legge, nel suo valore formale, per Ippia invece vale nel suo contenuto ciò̀ nella misura secondo cui s'accorda colla legge naturale. Onde mentre il Critone ci presenta Socrate morituro devoto alle leggi della patria e in atto di smentire il suo utilitarismo teoretico $\left.{ }^{16}\right)$, come una anticipazione vivente dell' esi-

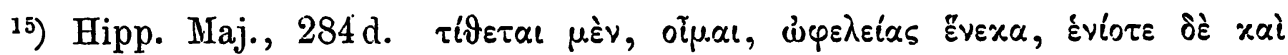

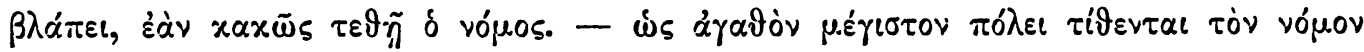

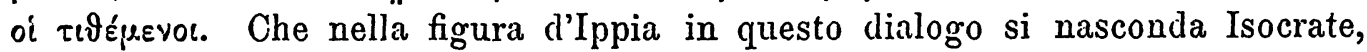
come vuole il Dümmler Akad. 56 ss., non so consentire, perchè è innegabile che alcuni tratti si riferiscono all' Ippia storico: come l'allusione al tóros parenetico d'Ippia (286a), e l'altra (285b s) agli studi astronomici e geometrici di lui, che in nessun modo si adatterebbero ad Isocrate.

16) Come ben rileva lo Ziegler, Gesch. d. Eth. I, 61. Philos. Monatsh. 1888 p. 451. Il tentativo dẹll' Heinze, Eudäm. in d. Griech. Phil. 1\$83, p. 752 
genza morale dell' imperativo Kantiano, Ippia riesce alla opposizione fra la legge positiva e la natura: poichè quella, che è un prodotto della storia, è nata per una volontaria convenzione umana, e non è che un precetto convenuto di comune accordo fra $\mathrm{i}$ cittadini e tradotto in iscritto ${ }^{17}$ ). Ma l'autorità sua gli pare venga meno quando coloro che l'han posta la riprovano e la mu$\tan 0^{18}$ ). Il segno delle leggi di natura, o leggi non scritte è invece il ritrovarsi di esse in ogni paese ${ }^{19}$ ) e perciò non sono opera umana ma divina ${ }^{20}$ ).

Gli scritti e l'insegnamente d'Ippia erano, come sembra, ispirati da questa dottrina, da cui esce il concetto cosmopolitico dei Cinici e degli Stoici ${ }^{21}$ ). Il discorso parenetico di Nestore al giovine Neottolemo su ciò che sia bello il fare, doveva, al pari dell' apologo di Prodico, presentare un quadro della semplicità della vita antica (Hipp. Maj. 286 A). Lo stesso apparisce dal proposito espresso in un suo frammento (presso Clem. Strom. VI, 624 A) di comporre dagli antichi poeti o prosatori, greci e barbari, un' opera attraente per varietà e novità; quella stessa forse che Ateneo (XIII, 609 a) cita col titolo ovva. $\omega \gamma_{\eta^{\prime}}^{22}$ ); e molto più da un altro, conservato da Stobeo ${ }^{23}$ ), nel quale egli afferma che la legge è in-

di dimostrare che gli ultimi atti di Socrate sono coerenti alla sua veduta utilitaristica mi sembra uno sforzo vano, come riconosce anche il Wundt, Ethik, 1886 p. 238.

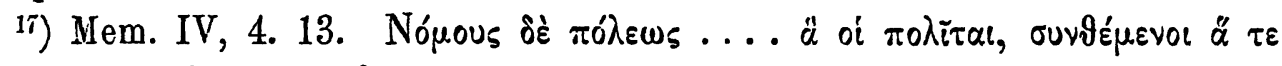

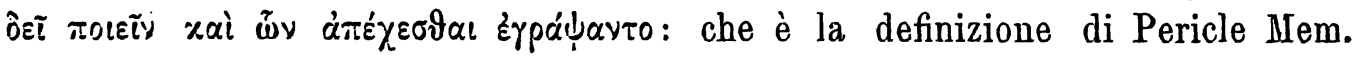
I, 2,42 .

18) Ib. 14.

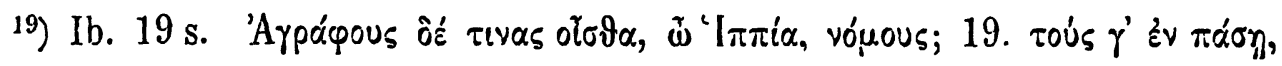
E้

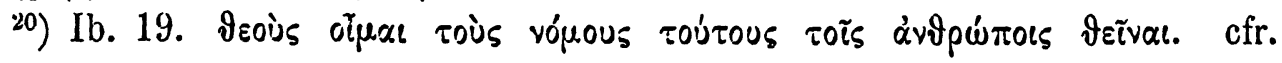
24, 25.

2) V. i frammenti d'Ippia in Müller Fragm. Histor. Graec. II p. 61 ss. Che le orazioni 75 e 76 di Dione Crisastomo sieno una elaborazione Cinica delle dottrine d'Ippia, come crede il Dümmler, Akad., 254, mi pare una ipotesi non dimostrata.

22) Il Müller (Fragm. II, 61) attribuisce invece questo frammento allo scritto

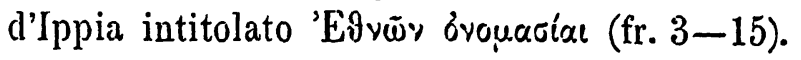

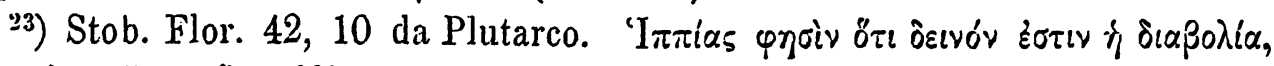

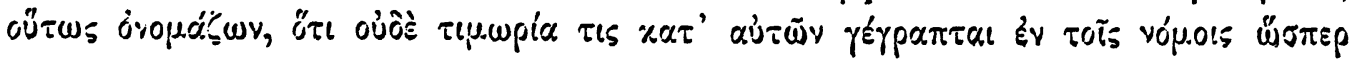


sufficiente e non provvede a tutto, mentre nessuna legge punisce la calunnin che è un male assai peggiore del furto, e contro cui non vi ha difesa. Ippia avverte che l'ordine morale è ben più ampio che di quello sottoposto alla sanzione delle leggi.

Questa teoria si riflette anche nella figura d'Ippia. La polimathia di lui, di cui ci parlano Senofonte e Platone, non si circoscrive ad ogni specie di composizione letteraria, si estende anche alle arti della vita. E nei giuochi olimpici, dinanzi alla Grecia, egli si vantava che non si desse arte di cui non fosse esperto, non solo fra le più elevate discipline ma fra le umili arti della vita ${ }^{24}$ ). Ora questa versatilità di lui, tanto ammirata in Sparta, è quella che distingue le società primitive, e che i fautori dello stato di natura propugnarono in ogni tempo; poichè in quello stato ogni individuo deve cercare da sè la maggior possibile cultura. Quanto più risaliamo nelle tradizioni antiche, tanto più vediamo che l'ideale del sapiente è quello d'uno a cui nulla è negato, che tutto sa, tutto può; mentre, come ben vide Aristotele, la divisione delle funzioni e degli uffici è condizione e insieme frutto, e in ogni modo segno del viver civile. Se Ulisse era $\pi 0 \lambda u ́ \tau p o \pi s \varsigma$, Pericle fu maestro solo nelle due arti, la politica e l'eloquenza, Demostene sarà solo di quest' ultima. È in fondo lo stesso ideale che rifiorisce nelle teorie educative di Rousseau e degli Enciclopedisti del sec. XVIII, che presentano un tipo di educazione fondata sulla sola natura ${ }^{25}$ ).

$\tau \tilde{\omega} \nu$ $\alpha \lambda \varepsilon \pi \tau \tilde{\omega} \nu$. Müller Fragm. Hist. Graec. II p. 63 fr. 14 . Allo stesso ordine d'idee in difesa d'un ritorno alla natura possiam ricollegare l'altro frammento i Fr. 7 (Ib. 62) secondo cui sembra che Ippia avesse notato come il nome di túpavvos (e con esso, s'intende, la cosa) era di recente fattura, ed era entrato fra i Greci all' età d'Archiloco. Questo ci spiega perchè Ippia avesse sosti-

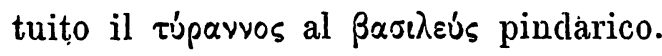

24) Cic. De Orat. III, 32. Plat. Hipp. min. 863 c. cfr. Philostr. V. Phil., 476.

${ }^{25}$ ) cfr. Benn The Greek Philos. I, 83, e Hildenbrand, Gesch. und Syst. d. Rechts- und Staatsphil. I, 1860 p. 77, i] quale del resto non coglie il vero affermando che la negazione del valore di ogni società e di ogni vita politica presso i Sofisti, derivi dall' individualismo comune ad essi. Questo è vero solo degli ultimi non dei più antichi Sofisti, che alla vita sociale contrapporgono una norma universale ricavata dalla natura. 
Non si deve credere però che Ippia sia un conservatore e che il suo pensiero miri ad un ritorno verso l'antica tradizione aristocratica; nè lo potremo aspettare da una teoria che fiorisce in un epoca di largo e intenso movimento democratico. In quanto anzi assaliva le leggi positive degli stati a cui contrapponeva come norma morale la natura, conteneva un germe di profondi rinnovamenti sociali, e se non ancora sovversiva, era almeno molto liberale. L'applicazione di quel principio doveva condurre a distruggere le ineguaglianze delle classi sociali su cui si fondava la vita antica, e il concetto della abolizione della schiavitù doveva esser potentemente alimentato dalla idea che questa sia contraria alla natura. Per questo la schiavitù è combattuta dai sofisti, e come Alcidamante, scolare di Gorgia, disse che gli Dei fecero tutti liberi e la Natura nessuno fece schiav $0^{26}$ ), cosi lo conferma la stessa critica di Aristotele, il quale espressamente combatte l'opinione di molti ai quali sembrava che il dominare gli altri sia un fatto contro natura, e che vi sieno schiavi solo per legge (vó $u$ ), ma non per natura ('ó́csl). Onde la schiavitù è opera non di natura, ma di violenza ${ }^{27}$ ). A questa corrente d'idee liberali e innovatrici se ne associava naturalmente un altra, avversa all' antica idea aristocratica delle nobiltà della stirpe, o dell' eùrýsveı; ed un sofista Licofrone affermava che la nobiltà del sangue è un nome vano e in nulla evidente $\left.{ }^{28}\right)$. Il ritorno alla natura non era dunque nè aspirazione all' antica vita delle aristocrazie greche, nè una teoria astratta da potersi paragonare alle dottrine dei sentimentalisti inglesi del sec. XVIII ${ }^{29}$ ), ma come la teoria dello stato di natura di Rousseau e degli enciclopedisti francesi mirava a scendere nella vita, e precorreva da vicino un profondo rinnovamento sociale.

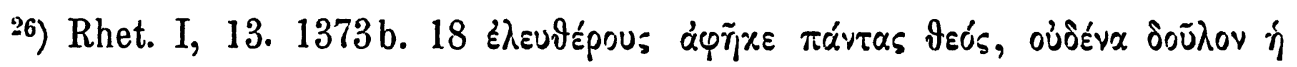
gíscls $\pi=\pi$ oin $\% \varepsilon \%$.

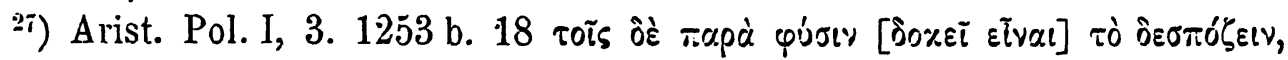

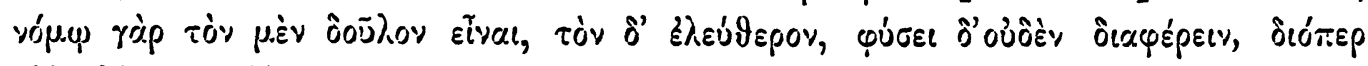
oủòè òizaıov Pícıv ráp. cfr. Oncken, Staatslehre d. Arist. II, $32 \mathrm{~s}$.

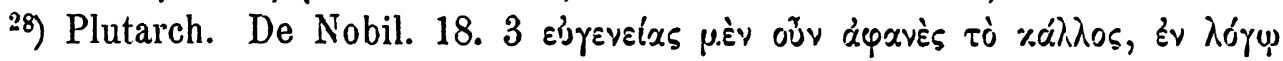

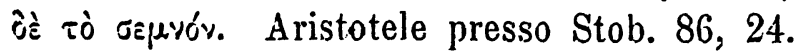

29) cfr. Leslie Stephen English Thought in the Eighteenth Century II, 1881 p. 449 ss. 
Questa critica della schiaviti come istiturione sociale, e sopratutto l'avversione alla idea aristocratica della nobiltà, piuttosto che da una reazione contro i pregiudizi sociali, in fatto muoveva da una corrente avversa alla scuola della natura, la quale avrebbe ben potuto riuscire alla giustilicazione della schiavitù e sopratutto dei privilegi nobiliari. Quel Licofrone che negava ogni valore alla nobiltà non era difatti seguace d'Ippia, ma, al pari d'Alcidamante, discepolo di Gorgia ${ }^{30}$ ); ed è quello stesso che secondo Aristotele,

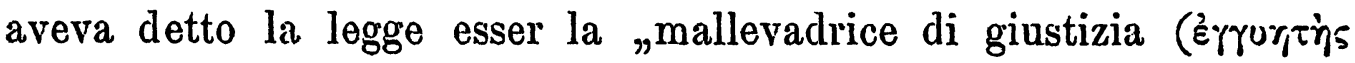
$\tau \tilde{\omega} \nu$ ôxai $\left(\omega \nu^{31}\right)$. Anzi Aristotele stesso, com' è noto, sosterrà la schiavitù come una condizione posta da natura ${ }^{32}$ ) e già Callicle nel Gorgia aveva detto che "per natura non è da uomo libero il soffrire ingiustizia, ma da schiavo, a cui è meglio il morire che il vivere, poichè non gli è lecito il soccorre a sè e ai suoi pari quando sia offeso e ingiuriato " ${ }^{33}$ ). Queste ardite dottrine non uscivano dunque della scuola dei partigiani della natura contro la legge sociale, ma dai fautori di questa contro la natura.

Ora codesta inversione dell' antitesi rilevata da Ippia per primo è quella che distingue il secondo gruppo di Sofisti (Gorgia e Protagora). Ma intanto il primo impulso a questo moto di pensiero liberale era venuto da coloro che distinsero nella vita umana il fondamento naturale, e ciò che è opera di consuetudine e di tradizione, e ne posero in luce $\mathrm{i}$ frequenti dissidi.

È naturale che $\mathrm{i}$ primi iniziatori di questo moto di pensiero non intravedessero le lontane consequenze sovversive a cui giunsero audacemente i sofisti della giovane generazione. Non sembra che Ippia volgesse la distinzione fra natura e convenzione sociale a difesa del dispotismo; piuttosto apparisce che egli e la scuola sua mirassero a dedurne una idea più conforme alle tendenze democratiche contemporanee, cioè l'idea cosmopolitica che precorre Anti-

30) Arist. Rhet. III, 3. Alex. Top. 209 e 222 (Zeller I', 960. 3).

31) Polit. III, 9. 1280 b. 11 (ed. Susemihl). Alex. Soph. Elench. 310. a. 12. Metaph. 533, 18. Bonitz.

${ }^{32}$ ) Polit. I, 4 e 5 . cfr. VII, 3. 1325 a, 28 . Oecon. I, 5.

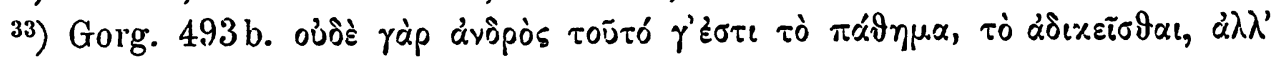

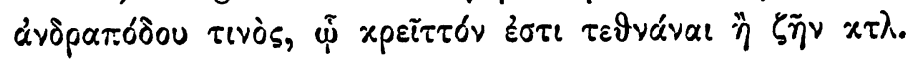


stene e la Stoa. Egli, il Rousseau del suo tempo, ha fede che la moralita si fondi non sul calcolo dell' utile proprio, frutto della convivenza civile, ma sopra le primitive benevole inclinazioni naturali. Ma intanto aveva cominciato a gettare il discredito sul vóuos e sull Ethos sociale, ad attaccare in nome della natura tutto quell' insieme di principi e consuetedini che si incarna nello stato; il quale apparisce come una finzione sociale, dove le norme del dritto non sono che una permanente cospirazione di pochi contro a ciò che la natura dette a tutti gli uomini; cospirazione tanto più pericolosa in quanto che si nasconde sotto la veste del diritto ed è di fatto come sancita dello stato.

Ora codesta critica di uno dei termini della antitesi non poteva rimanere senza frutto. Basterà che altri volga le stesse armi della critica contro la natura come norma della vita morale, perchè da questa "mutua strage" delle due opposte direzioni, per dirlo con Sofocle, si traggano le inevitabile conseguenze negativè, e si elevi a solo giudice e misura anche nella vita morale e sociale l'individuo senza limiti e senza freni. E mentre alcuni nel senso di Callicle nel Gorgia, ritornando alla idea d'Ippia che sola la natura è norma della vita, troveranno nella natura stessa la giustificazione della violenza e del dispotismo, perchè in natura impera sola la forza; altri invece troveranno che la stessa vita sociale è la perfetta, sebbene dissimulata, applicazione di questo principio naturale, giacchè le leggi sono opera dei più potenti, che cospirano contro il bene di tutti. Ma a codeste estreme conseguenze non si arriverà se non per via della seconda fase del pensiero sofistico, cioè non prima che si sia formata una corrente di dottrine contraria a questo ritorno all' idea della natura. Il distaccarsi del pensiero da quella idea, prima che si giunga a contrapporre la sfera subiettiva all' ordine obiettivo delle cose, si manifestava da un lato nella sfiducia crescente verso la speculazione fisica, dall' altro nel contrapporre alla natura come principio morale, l'opera del costume e della vita civile. Questo è il terreno comune in cui si muovono, giungendovi da opposte vie, Gorgia e Protagora.

Che Gorgia abbia atteso, forse nella sua gioventù, agli studi fisici, e che a questo periodo sia dovuta la teoria dei colori che 
gli è attribuita nel Menone platonico, e si collega alla fisica d'Empedocle, è reso assai probabile oggi dal Jiels ${ }^{34}$ ). A questo periodo del suo insegnamente nel quale ebbe parte anche la Fisica segui, sccondo la verisimile ipotesi dal Diels, grazie forse alla critica di 'senone, un periodo di dubbio, il cui frutto fu appunto lo scritto "intorno alla natura". Ora che lo spirito di esso fosse la negazione non solo della antica fisica iu generale, ma più specialmente della

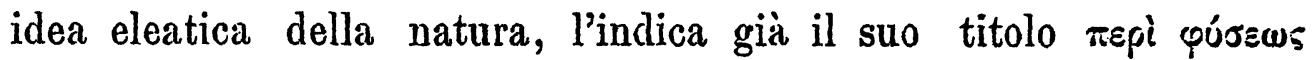

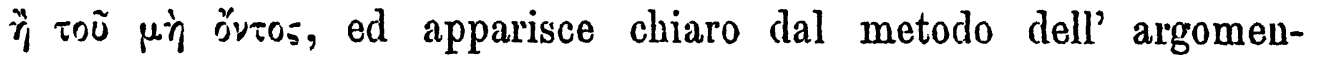
tazione, e dalla distribuzione delle tesi ${ }^{35}$ ). In questa critica d'ogni conoscibilità del vero si spinse fino all' antitesi della tesi parmenidea, giungendo al resultato d'un estremo Nihilismo e agnosticismo. La distinzione che il Grote ${ }^{36}$ ) attribuisce a Gorgia fra la sostanza 0 il sostrato Noumenico, che secondo il critico inglese avrebbe solo negato agli Eleati, e il fenomeno che solo ammetteva, è straniera a tutto il pensiero antico prima di Platone, e tanto più a quello di Gorgia; poichè non solo le fonti antiche non accennano a questi limiti della sua negazione, ma ci offrono tali dati da poterla escludere. Nella ipotesi del Grote la argomentazione gorgiana avrebbe logicamente dovuto cominciare della seconda proposizione, cioè non che nulla sia, ma che l'essere vero e. sostanziale non è conoscibile $^{37}$ ).

E vero però che l'ultima prova della tesi di Gorgia confina, - meglio combacia perfettamente col relativismo di Protagora, L'incomunicabilità della conoscenza poggia tutta per Gorgia sulla ragione del più rigido individualismo sensistic $0^{38}$ ). La negazione gorgiana lascia solo l'adito alla sensazione individuale, che Prota-

34) Diels, Gorgias und Empedokles. Sitzungsber. d. Berl. Akad. 1884 , p. 343 ss.

35) In qual modo più precisamente la dialettica negativa di Gorgia scaturisca dalle contradizioni della scuola eleatica l'ha posto in chiaro assai felicemente il Bäumker. Neue Jahrb. f. Philol. 1886, p. 560 s.

36) Grote, Histor. of Greece VI, 70.

${ }^{37}$ ) cfr. le osservazioni dello Zeller I ${ }^{4}, 987$.

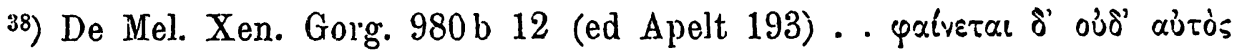

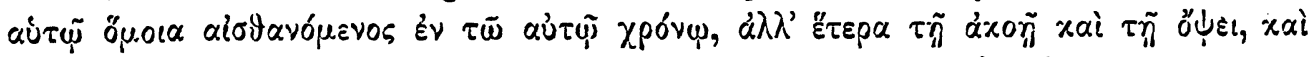

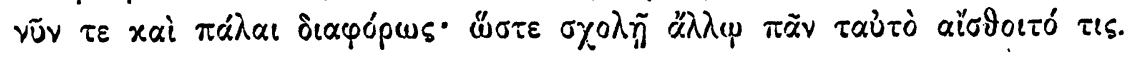


gora poneva a misura e centro di tutte le cose. Esclusa la possibilità d'una conoscenza obiettiva della natura, non rimane che l'opinione che qualchecosa vi sia 0 che le cose sieno in quanto e come appariscon $0^{39}$ ); e poichè codesto yaíveơal, se si fa una ésatta riduzione degli elementi conoscitivi, ha le sue ultime radici nel soggetto così questo è il solo che sornuoti in tanto naufragio. D'altra parte la negazione teoretica si converte in una affermazione pratica; poichè alla praxis basta la semplice ôó́́ $\alpha$, la quale si può trasformare in convinzione per via della Retorica ${ }^{40}$ ). Gorgia rinunziando quindi alla yóoıs, trovava aperta la via all' arte del persuadere, o meglio ad una delle tre arti del persuadere che si distinguono nell' Encomio di Elena- che porta il suo nome (Helen. $\S 13$ ): ed egli stesso vuol esser considerato non già come un Sofista, bensi come un Retore (Plat. Gorg. 449 A).

Tutto questo ci fa intendere quale posizione abbia preso Gorgia difronte all' obiettivismo di quelli che si potrebber dire i fisiocrati del suo tempo, e al naturalismo precedente e contemporaneo. La tecnica del suo stile, così artificioso e fiorito ${ }^{41}$ ), mostra di per sè quanto egli si tenesse lontano da tutto quello che è semplicità di natura. E se non abbiamo dati per affermare ch'egli si pronunziasse nella questione intorno al rapporto fra la púots e il vónos nella vita umana $^{42}$ ), pure il suo punto di vista ci fa intendere come egli, anche per questo rispetto, si ravicicinasse alla tendenza di Protagora.

Che il relativismo di Protagora nella teoria della conoscenza debba essere inteso come un rigoroso subbietivismo o individualismo, secondo che vien presentato nel Teeteto da Platone che lo ricollega alle dottrine degli estremi eraclitei (Cratilo), è stato bensi negato dopo il Grote ${ }^{43}$, negli ultimi anni da alcuni critici, e sopratutti dal

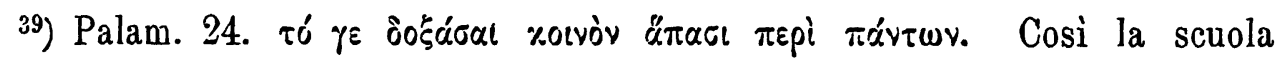
eleatica finiva al punto di dove era partito Senofane. fi. 14. Karsten . oóóxos

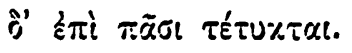

40) Diels, Sitzungsb. d. B. Ak. 1884 p. 360 s.

4) Sul roprix́sely cfr. Diels. Op. cit. 364 ss.

42) Come fa il Benn, Greek Philos. I, 96.

43) Grote, Plato and the other comp. of Socr. II, $325 \mathrm{ss.}$ 
Laas c dall' IIallbfass ${ }^{\text {t4 }}$ ); i quali nella celebre formula di Protagora che fa l'uomo misura di tutte le cose non trovano espresso che tale sia l'individuo o il soggetto singolo, ma solo che l'uomo in generale sia il centro e la misura dell' universo. Poichè non è qui il luogo di discutere a fondo questa interpetrazione recente, e dimostrare che l'esposizione platonica, come noi ancora fermamente crediamo ${ }^{45}$ ), presenti ben distinte le parti originarie della dottrina storica di Protagora, dagli svolgimenti speculativi che Platone ne ricava con magistrale e insuperata potenza, giova al nostro fine solo rilevare come il significato generico ed ampio attribuito a quella formula, se è inesatto nel rispetto teoretico, ben risponde invece alle dottrine etiche di Protagora. Nella controversia sui rapporti fra la natura e il costume o la vita civile, egli prende parte per questa, e rivolge le sue armi contro i fautori della natura, mostrando ad essi che quella costanza ed universalità che le attribuiscono rispetto a ciò che è opera e artificio umano, non è che una illusione, poichè anche la natura è in preda a una vicenda che mai non resta, e tanto ne sappiamo per quanto ce ne attesta il nostro sentire ${ }^{46}$ ). Che il discorso sulla virtù civile tenuto da Protagora nel dialogo platonico che porta il suo nome sia una critica delle teorie di Prodico e sopratutto d'Ippia, ce lo fa pensare già l'allusione ironica a quei sofisti che riconducono i giovani allo studio delle arti e delle scienze fisiche e alla quale Platone aggiunge le parole „e insieme diede una occhiata a Ippia ${ }^{47}$ ); e il fatto stesso che Prodico e Ippia sono presenti a questa allocuzione. La quale è poi in sè stessa tutta una confutazione delle teorie anticivili e naturalistiche di Ippia.

Prima che Prometeo, secondo il mito di Protagora, rapisca

44) Laas, Idealismus und Positivismus 1879 I. p. 26 ss. Halbfass, Die Berichte des Plat. u. Arist. über Protag. Leipzig 1882. cfr. Laas in Vierteljahrs. f. wiss. Phil. 1884 p. 479-97.

45) Vedi la critica acuta della tesi dell' Halbfass in Natorp, Forschungen, 1884 p. 1-62. Meno risoluto si mostra il Sattig in Zeitschrift f. Phil. 1885 p. 275-320, 1886 p. 1-34. cfr. B. Münz, Protagoras und kein Ende. Zeitschr. f. Phil. 1888 p. 109-125. cfr. L. Stein, Die Erkenntnisstheorie der Stoa. Berlin 1888.

46) Benn, The Greek Philosophers II, p. $87 \mathrm{~s}$.

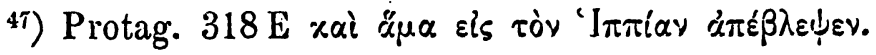


il fuoco ad Hefestos e a Minerva l'arte del sapere, non vi era vita civile (321 d). Fatto partecipe della sorte divina, l'uomo riconosce gli Dei (allusione questa e critica, io credo, della teoria di Prodico) e articola le prime voci. Ma non è ancora sorta colle città la vita civile $(323 \mathrm{~b})$. Siamo ancora allo stato di natura che Protagora „ci rappresenta come un bellum omnium contra omnes". „Si danneggiavano fra loro non avendo l'arte civile, talchè di nuovo dispersi perivan $\left.0^{(48}\right)$. Giove quindi per salvare gli uomini manda

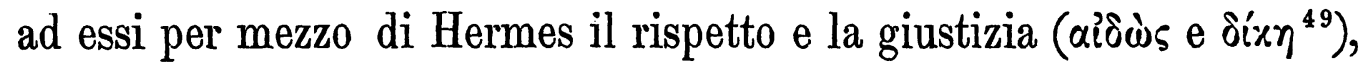
dalle quali virtù nascono gli ordini civili, e l'amicizia fondamento del vivere sociale; poichè, come dice, non nascerebbe le città se di queste due virtù tutti quanti non partecipassero ${ }^{50}$ ).

Nè si creda, come fa l'Harpf ${ }^{51}$ ), che Protagora fondi perciò la sua etica sopra una specie di senso morale di natura, al modo dell' Hutscheson. In questo egli differisce del moralista inglese che mentre per questi il moral sense è natural sense, per lui questo senso è formato colla vita civile. Ora a questo problema delle origini della virtù civile, se ne collega per Protagora intimamente un altro che concerne la natura e l'esistenza stessa della professione dei sofisti, maestri di virtù. Se questa, come credeva Ippia, è un dato naturale innato, a nulla giova ed è assurdo l'insegnarla. Perchè questo insegnamento sia possibile, è necessario dimostrare

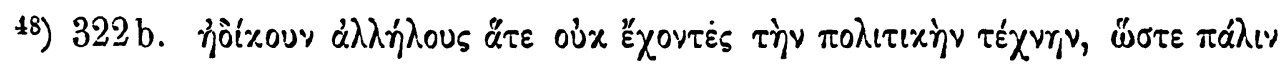

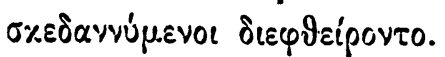

49) cfr. Bonghi. Note al Protagora 1882 p. 255-61. L. Schmidt, Eth. d. alten Griech. I, p. 168 ss. 1882. Non è stato avvertito che io sappia, che già Protagora (323c) prima di Aristotele aveva riconosciuto che la distribuzione degli uffici è condizione essenziale della vita civile.

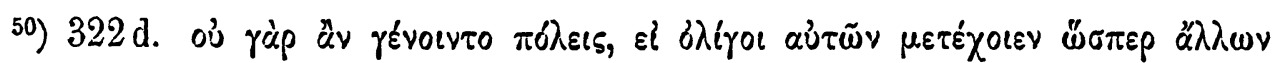

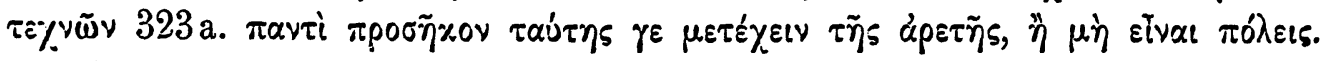

51) Harpf, Die Ethik d. Protagoras. Heidelberg 1884 p. 14s., il quale s'inganna facendo di Protagora un fautore del naturalismo etico. Lo prova la stessa osservazione ch'egli fa p. 15 sulla chiusa del mito di Protagora, che rivela le tendenze democratiche di lui. Se Hermes dà a ciascuno le tendenze etico-sociali, è la vita civile dunque che tutti aguaglia in perfetto comunanza. Del resto la tesi dell' Harpf, che s'accorda anche colla interpetrazione del Dümmler Akademika, 251, è confutata da tutto quello che vien dopo nel discorso di Protagora. 
che la virtì sia acquisita, sia frutto d'esercizio, d'una disciplina, o che l'animo vi si possa educare. Ed ecco quello che Protagora dichiara di voler dimostrare a Socrate ${ }^{52}$ ). $E$ in primo luogo ne è una prova per Protagora la pena pubbiica, ch'egli con mirabile penetrazione ${ }^{53}$ ) considera come un motiva d'intimidazione per l'avvenire piuttosto che come punizione per un misfatto trascorso, poichè non renderebbe mai il fatto non avvenuto ( $324 \mathrm{~b}$ ); la qual cosa è segno che, secondo l'opinione comune, la virtù è frutto di

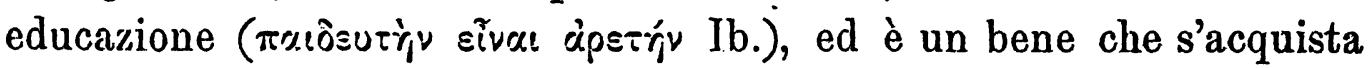
per via di diligenza, d'esercizio e d'insegnamento ${ }^{54}$ ). Su questo punto Protagora è interamente d'accordo con Socrate, nè dubita, proseguendo nel suo discorso, di rilevare come la vita privata e la vita pubblica del cittadino greco sia tutta un insegnamento di virtù $(325 \mathrm{~d}-325 \mathrm{E})$ e un efficace sistema di educazione.

La quale osservazione gli porge il destro di ritornare ancora contro la teoria dello. stato di natura, paragonando la vita civile a quella di uomini che non hanno nè educazione, nè tribunali, nè leggi nè forza alcuna che li costringa interamente a curar la virtù, ma siano una specie di selvaggi, come quelli che un tempo Ferecrate il poeta aveva presentati alle Lenee ${ }^{55}$ ). Con fine ironia Protagora rassomiglia gli uomini, quali se li fingevano Ippia e $\bar{i}$ fautori dello stato di natura, al coro dei misantropi in quella commedia (ib.); nè forse è lontano dal vero il supporre che in questa stessa commedia, rappresentata nel 421 , codesto coro dei misantropi che, fuggendo la vita civile nelle solitudini incontravano i selvaggi, fosse una caricatura di questa scuola politica dei Rousseau e dei Diderot di quel tempo, qui combatutti da Protagora. Si ponga

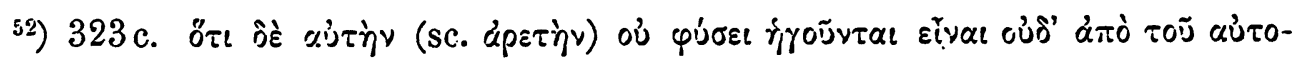

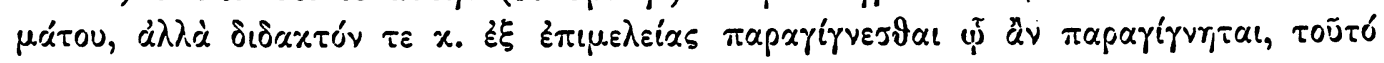

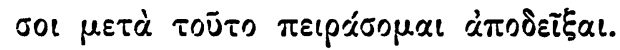

53) Grote, Plato and the other comp. of Socr. II, p. 41. Schmidt op. cit. II, 258.

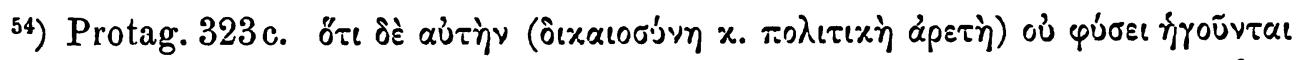

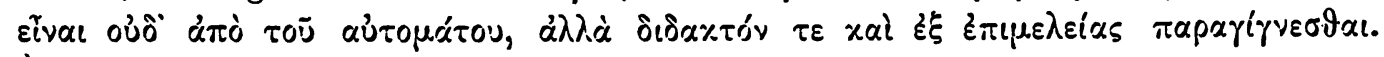
É facile pensare come queste parole colpiscano Ippia, che è presente. Ib. d.

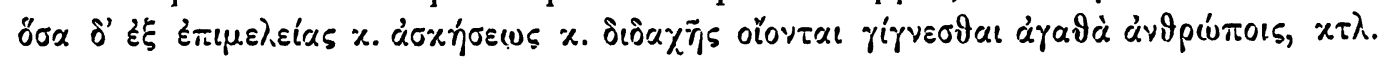

55) 327 c. d. 
mente al fatto che, come tutto fa credere, il mito di Protagora nel dialogo platonico, è probabilmente un estratto, 0 almeno è scritto nello stesso ordine d'idee di quello che sappiamo aver composto

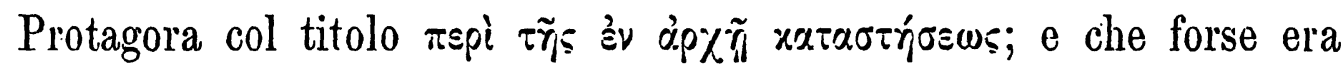
una critica delle teorie correnti sullo stato di natura ${ }^{56}$ ).

Cosi intesa ad ogni modo l'orazione di Protagora, come una confutazione delle teorie dei naturalisti contemporanei e come una apologia del vóuos e della $\pi o_{i} \iota s$, sparisce ogni differenza fra la teoria da lui espressa in quel dialogo, e quella che è posta in bocca sua nel Teeteto platonico, che a molti critici sono parse cosi inconciliabili, da doversi o negare la fedeltà storica della prima, come ha fatto lo Steinhart ${ }^{57}$ ), o quelle della seconda come ha fatto lo Schanz ${ }^{58}$ ). V'è anzi un intimo nesso logico fra l'una e l'altra, e un fondamento comune in ciò che solo lo stato e la legge civile determina ciò che è bene, e che il giusto, come direbbe Socrate che fin qui è d'accordo con Protagora (Xenoph. Mem. IV. 4), è ciò che è legittimo, o legale.

Le due dottrine morali poste in bocca a Protagora s'integrano l'una coll' altra. Nel Protagora è rilevata la origine della virtù umana nella vita civile, e dimostrata la possibilità d'insegnarla e di svolgerla colla educazione. La vita sociale e lo stato vi era presentata come la vera forza educatrice dell' individuo. Protagora non cercava, come Socrate, che cosa la virtù sia; gli bastava il trovarla già fissata e quasi sancita dall' autorità del re Nomos, come ben nota il Grote ${ }^{59}$ ). Ora la difesa che Protagora fa di sè stesso nel Teeteto $(166 \mathrm{~A}-168 \mathrm{C})$ dimostra come lo stato

56) Su questo titolo Frei, Quaestiones Protagoreae, Bonn 1845 p. 182 ss. Köstlin, Gesch. d. Ethik I, 1887 p. 221. Non so perchè lo Zeller crede il mito di Protagora derivato piuttosto dello scritto di lui sulla Politia.

57) Steinhart, Platon's Werke J, p. 422.

${ }^{58)}$ Schanz, Beiträge z. vors. Philos. p. 110. cfr. invece Peipers, Erkenntnissth. Platons p. 548. Archer-Hind, The Theaetetus of Plato, Oxford 1883 p. 91. Steinhart, Op. cit. III, 36. La conciliazione a cui riccorre lo Schuster, Her. von Eph. p. 323 mi pare un sottile ma vano espediente.

59) Grote, Plato II, 72 s. Questa fede di Protagora nella legge politica è un altro punto di contatto, meno avvertito, con Eraclito, di cui si ricordi il frammento 100 (Byw.). 
si faccia norma della vita morale per mezzo delle leggi; e che in questa la vera misura è la legge o come egli la chiama la dóćs $\pi$ rélews. Ciò che è giusto ed onesto è tale perchè determinato

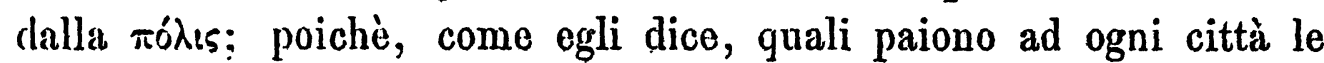
cose giuste ed oneste, così anche sono per essa finchè le reputi tali $\left.{ }^{60}\right)$. In quanto dunque ogni stato determina ai suoi cittadini ciò che ò legittimo o giusto, in tanto, come dice altrove, non si dà uno più saggio dell' altro, nè da privato a privato, nè da città a città $\left.{ }^{61}\right)$. Se non che come nel Protagora aveva detto che non ogni piacere è bene, così anche delle leggi civili le une, se non più vere, possono ben essere più utili agli stati delle altre. E qui appunto entra l'opera del sapiente, il quale a guisa del medico che raddirizza le sensazioni dell' ammalato e le rende migliori (167 A ss.), nel campo morale e politico sa per mezzo della educazione, corregere e migliorare le disposizioni, e agli stati suggerire ciò che è migliore e più utile $(172 \mathrm{AB})$, riuscendo così a far mutare l'opinione dello stato o la legge (167C-177D). Entro la cerchia della Poli, e finchè è in vigore una legge, la giustizia (óvacov) ha un valore assoluto. Ma non lo ha in realtà, in quanto poggia sopra un dato naturale ${ }^{62}$ ). L'elemento morale si risolve

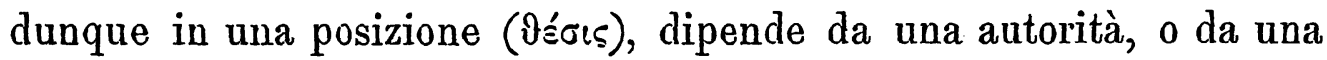
convenzione politica ${ }^{63}$ ).

Alla stessa tendenza si collegavano, come sembra, le dottrine grammaticali e filologiche di lui. Che anche nella questione così

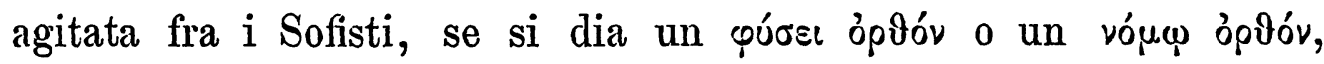
Protagora propugni quest' ultima opinione, e non già la prima come crede lo Schuster ${ }^{64}$ ), risulta non solo dal Cratilo platonico, dove

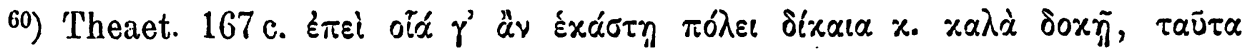

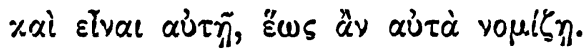

Non so come l'Archer-Hind, The Thaet. of Plato p. 98 trovi cosi naturale il passaggio dal relativismo individuale nell' etica accennato al $157 \mathrm{~d}$. a questo relativismo politico.

61) 168 b. 172 A. cfr. Münz, Zeitschr. f. Philos. 1888 p. 121 s.

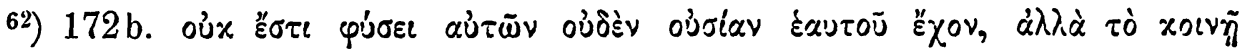

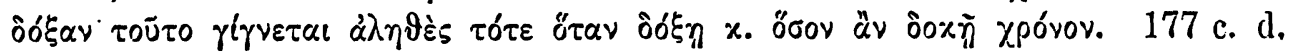

63) Laas, Idealismus und Positivismus II, p. 2 ss. 1882.

${ }^{64)}$ Schụster, Heraklit. p, 323 ss. 
l'autorità di Protagora viene appunto associata alla tesi d'Ermogene, cioè dell' origine convenzionale del linguaggio, ma ancora dal Protagora, nel modo con cui sopra è stato interpretato il mito contenutovi. Come Protagora vi ha dimostrato in forma narrativa che la giustizia non è da natura ma d'origine civile, cioè quello stesso che è affermato dal Teeteto $(167 \mathrm{C})$, così le prime voci nascono quando l'uomo, uscito dallo stato selvaggio di natura, si avvia a vita civile ${ }^{65}$ ). La dottrina che dà al linguaggio un fondamento obiettivo nella natura non poteva esser quella di chi ammetteva che ciascuna cosa è a ciascuno come gli appare.

E però vero che questa specie di normalismo esteriore in morale mal s'accorda col soggettivismo conoscitivo di Protagora; e noi ci sentiremmo tentati, per amore di coerenza, ad interpretare la formula dell' homo mensura al modo del Laas e dell' Halbfass, attribuendo a Platone l'interpetrazione strettamente individualistica di essa, se troppe gravi ragioni non ce ne dissuadessero. Piuttosto diremo che Protagora non osò applicare ai fatti morali lo stesso criterio audace della subiẹttività, 0 non ne misurò le conseguenze ${ }^{66}$ ). Una tale riserva rispetto all' ordine pratico è un fatto che si ripete bene spesso nelle storia del pensiero umano; e il costruire il mondo morale sopra un fondamento diverso da quello su cui poggia l'ordine conoscitivo, 'come è ben noto, è anche la posizione del Kantismo, la quale ben si può ravvicinare a quella di Protagora ${ }^{67}$ ). Ora questi a cui, come sofista, stanno sopratutto a cuore gl'interessi pratici, come prova il Protagora e la stessa sua difesa nel Teeteto, trova anche nel subiettivismo un elemento positivo che può esser principio d'una teoria etica e fa sentire alla scuola dello stato di natura che la vita morale sussiste di per sè perchè fattura dell' uomo e opera propria dello spirito.

Ma se non vi ha una connessione intima vi ha almeno una

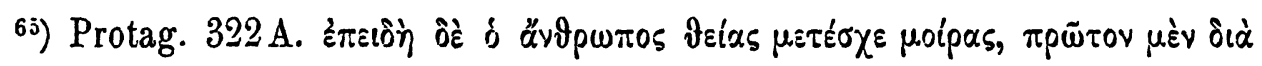

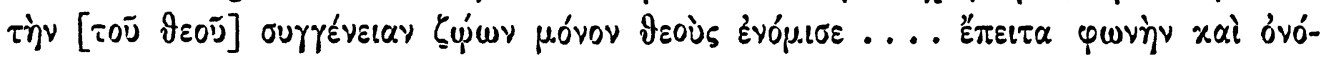

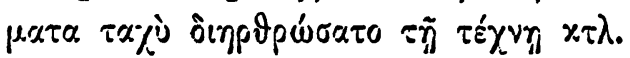

66) Frei, Quaest. Protag. p. 113. Benn, Greek Philos. I, p. 88. Münz, Zeit. f. Phil. 1888 p. 122 ss.

6i) Harpf, Ethik d. Protag. p. 40-57. 
corrispondenza esteriore quasi di simmetria fra il normalismo morale c il subicttivismo teoretico di Protagora; poichè la relatività del conoscere fit riscontro alla relatività delle norme morali secondo le diverse legislazioni degli stati e secondo i diversi tempi. E sopratutto s'accordano in questo, che ambedne son dirette contro la teoria della natura come norma morale. Quanto più anzi è incoerente la riscrva di Protagora nell' applicare all' etica l'estremo individualismo, tanto più è evidente in lui la tendenza ad accentuare contra Ippia, l'idea del Nomos e della Polis, e l'inclinazione alla statolatria, e a propugnare, egli l'umanismo del suo tempo, una educazione classica contro l'educazione scientifica seguita dal suo rivale. Mentre Ippia raccomanda il ritorno alla semplicità della natura, Protagora il democratico ha fede nell' educazione e nella vita sociale. Non senza ragione Pericle, come pare, aveva affidato a lui, autore d'un libro sullo stato, di dar leggi alla città di Thurii ${ }^{68}$ ).

Cosi la vena scettica di Protagora che muovendo della teologia, e investendo le discipline fisiche (Metaph. III, 2, 998 a. 3. Soph. 232 d), si estendeva ad ogni scienza, si era poi arrestata dinanzi ai problemi morali, la cui soluzione egli trovava già data nelle leggi positive per ogni stato. E, come appare da Aristotele ${ }^{69}$ ) non aveva estesa la regola del far migliore il discorso peggiore all' ordine delle idee morali. La misura delle cose non è il volere degli Dei la cui esistenza è incerta (D. L. IX, 51, Thaet. 162d.), non uno stato ipotetico di natura, ma solo il soggetto quanto al conoscere, solo la legge quanto all operare. Gorgia, mostrando le contradizioni intime della idea della natura, aveva intitolato il

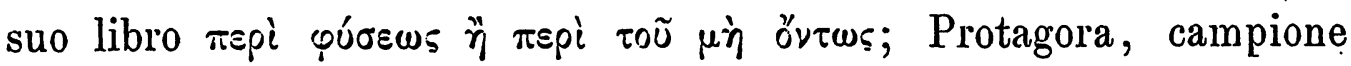

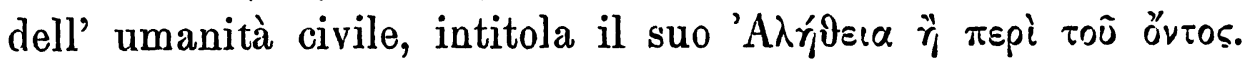

In questa corrente d'idee Protogora fu seguito da una folla d'altri sofisti. Licofrone che, come dicemmo, aveva chiamata la legge la „mallevadrice della giustizia“, Eveno di Paros che aveva scritto sulla virtù umana e politica ${ }^{70}$ ), tutti si collegano, come pare,

68) Eraclide Pontico presso Diogene IX, 50.

69) Rhetor. II, 24.

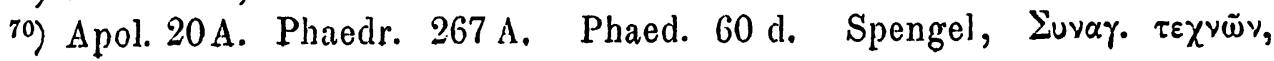


a questa teoria, che troviamo riprodotta dal grande storico del tempo. L'effetto che queste idea ebbero sul pensiero di Tucidide ci è manifesta nell' introduzione della sua storia. L'antichità è da lui pure presentata come una storia di barbarie, di solitaria e selvaggia violenza, e solo in un periodo assai recente Atene da questa oscura condizione è uscita, per lui, alla luce e alla gloria del suo tempo $\left.{ }^{71}\right)$. E anche nella orazione funebre che egli pone in bocca a Pericle la gloria leggendaria d'Atene, così celebrata dai logografi e dagli oratori, è appena accennata con fuggitiva allusione, e tutta la luce è raccolta sulla presente vita civile ${ }^{72}$ ). Da Eschilo e da Erodoto, il poeta e lo storico delle origini, siamo passati ad un realismo politico che da un lato s'accordava colle nuove dottrine della scuola di Protagora, e dall' altro rispondeva alle tendenze del tempo che piegava a democrazia.

C'era senza dubbio nella teoria del Nomos sovrano (o della

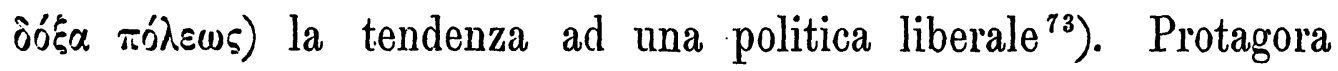
non era un conservatore, perchè il Nomos ch' egli voleva non era una immutabile tradizione; bensi una creazione umana, capace di mutamento e di perfezionamento. C'era nella dottrina di lui quella stessa tendenza che penetrava la politica moderatamente liberale di Pericle, e che, come suole, apparve invece conserva-

92 Bergk., Lyr. Graec. p. 474-79. Ueberweg-Heinze, Grundriss $1^{7}$, 1886

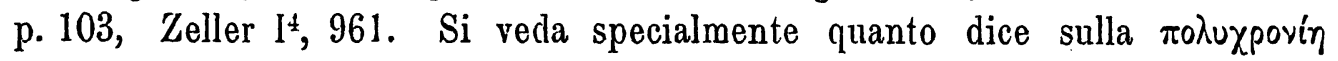
$\mu . \varepsilon \lambda_{\varepsilon} \tau \eta$ nel Fr. 9 (B.) presso Arist. Eth. Nic. VII, 11. Ib. 7.

7) Thuc. I, 2. 2. Si confronti questo passo col Protagora 322 b. cfr.

73) Thuc. II, 36. cfr. Zeller II', 23. Benn, Greek Philos, I, 90. Ziegler, Gesch. d. Eth. I, 1882 p. 273.

73) Come nota anche il Guggenheim, Die Lehre vom apr. Wissen in d. sokrat.-plat. Philos. Berlin, 1885 p. 70. Benn, The Greek Philos. I, 92. Alla influenza che ebbe la teoria di Protagora sulla sovranità del Nomos si può ricondurre, e non già al púvaı bpò̀y *. ócxaloy di Eraclito, come vuole il Lommer, Jahresber. d. Studien-Anstalt Metten 1879 p. 33, il concetto di Euripide.

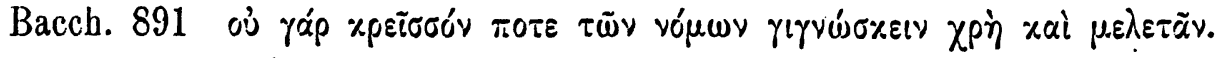

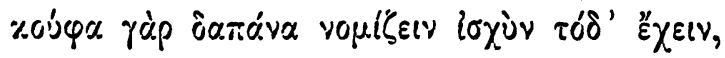

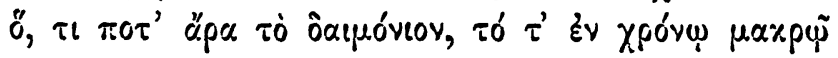

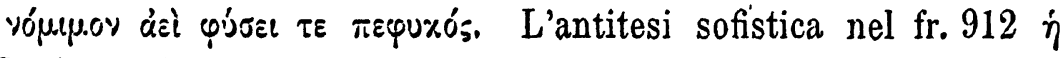

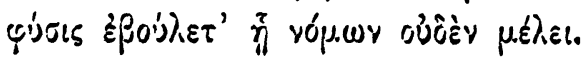


trice più tardi al contemporanei d'Alcibiade. Un esempio rilevante di corlesto conflitto fra l'idee politiche della giovine generazione $e$ la politica ispirata dalle idee di Protagora, e del passaggio dalle uno alle altre è il dialogo fra Alcibiade e Pericle, il tutore di lui, nei Memorabili (I, 2, 41-47) sulla idea della „legge". Pericle, com' è noto, familiare di Protagora, vi mostra ancora una fede in-

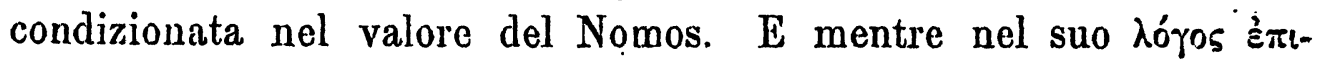

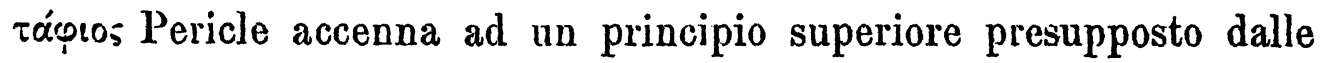
leggi positive ${ }^{74}$ ), nel luogo senofonteo Alcibiade, amico forse di Callicle il sofista ${ }^{75}$ ), lo costringe a riconoscere che la legge è un precetto che vale solo se vi è il libero consenso o la persuasione di coloro a cui è imposto; senza questo non è legge ma illegalità (àvou' $(\alpha)$ o violenza ( $\beta{ }^{\prime}(\alpha)$, da qualunque parte essa venga, o dalla tirannide di un solo, o dalla oligarchia o dalla massa popolare $\left.{ }^{76}\right)$. Così si comincia a discutere e quindi anche a scuotere la fede nella sacra autorità della legge, e lo spirito razionalistico insinua quel dubbio che via via andrà mettendo radici più profonde nelle menti e negli animi.

Messo una volta su questa via lo spirito razionalistico non poteva arrestarsi finchè non fosse giunto alle conseguenze più sovversive e antisociali. E vi giunsero audacemente i sofisti della nuova generazione le cui dottrine radicali sono largamente e senza ritegno propugnate da Callicle nel Gorgia, e da Trasimaco nella Repubblica di Platone. Le quali teorie, professate dai due sofisti o affiliati alle scuole dei sofisti, non costituiscono già, come si suol credere ${ }^{77}$ ), una sola forma di dottrina, e il Grote ha ragione di richiamare l'attenzione sulle loro differenze ${ }^{78}$ ); ma sono piuttosto

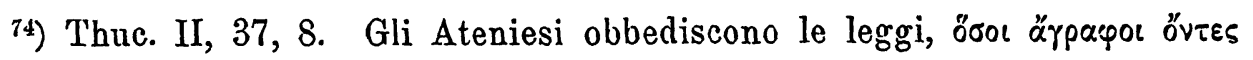

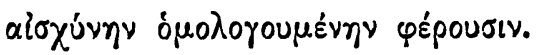

75) Perchè come ha osservato il Köstlin, Gesch. d. Eth. I, 241, Callicle è nominato due volte nel Gorgia $(481,519)$ insieme ad Alcibiade.

76) Xenoph. Mem. I, 2, 45.

77) Vedasi per tutti lo Zeller I, 1009, 5, il Köstlin 0. c. I, 232 s, e ora anche il Windelband, Gesch. d. alten Philos. 1888 p. $72 \mathrm{~s}$.

${ }^{78}$ ) Grote, Hist. of Gr. VI, 89. Vi accenna anche l'Hildenbrand, Geschichte u. Syst. d. Rechts- und Staatsphil. I, 1860 p. 70-74. 
differenze di origine che di resultato. Muovendo dai principii delle due opposte scuole, della natura contro le legge, e della legge contro la natura, riuscirono all' apoteosi della assoluta sovranità dell' individuo, o del dispotismo, o alla giustificazione dell' anarchia sociale. Avevano in fatti di comune almeno questo: che ambedue le scuole, contrapponendo i due termini, della natura e della legge, rompevano l'unità della vita morale, e ne mettevano in luce l'intima contradizione; ed erano così ambedue antitradizionali. Il principio d'Ippia 'che solo la natura, non la legge sia norma della vita, poteva facilmente piegarsi ad un senso non veduto nè voluto da lui. Poichè la condizione che natura pone non è già l'uguaglianza, com' egli credeva, ma la prevalenza del più forte sul più debole. In questo bellum omnium contra omnes, come dice Hobbes, 0 in questa lotta per l'esistenza, come direbbe un darwinino d'oggi, solo ai forti il trionfo e la vittoria. Se dobbiamo ritornare alla natura, e respingere la legge 0 il diritto che circoscrive la azione di ciascuno rispetto agli altri e tiene a freno i più forti, la conseguenza logica è la giustificazione del dispotismo.

Questo è difatti il ragionamento di Callicle nel Gorgia ${ }^{79}$ ).

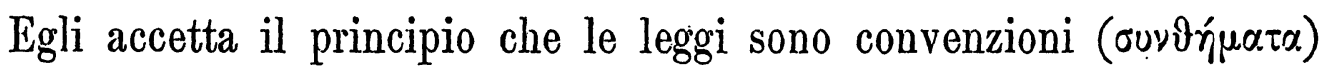

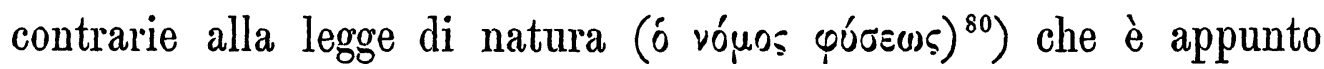
la massima d'Ippia. Ora nella natura chi ha la forza ha il diritto (ó čp $\chi \omega v)$, ed è giusto quello che egli vuole e fa, talchè per natura sia meglio il fare che il soffrire ingiustizia. Ma come

${ }^{79}$ ) Il rapporto fra la dottrina di Callicle e la scuola di Ippia apparisce nou solo dal modo da cui è espressa l'antitesi fra la natura e la legge (Gorg. $482 \mathrm{E}$ ), ma anche dal fatto che Callicle ripete a Socrate quella stessa difficoltà che egli dicesse sempre le stesse cose (Gorg. $4.90 \mathrm{E}$ ), che troviamo fatta appunto da Ippia. Xen. Mem. IV, 4, 6. Questa rispondenza vedo ora notata anche dal Dümmler, Aliademika, $252 \mathrm{~s}$.

${ }^{80}$ ) Gorg. $482 \mathrm{E}, 483 \mathrm{~b}$. E, $492 \mathrm{C}$. Che vi fossero due gruppii contrari di Sofisti, i fautori del costume, e i fautori della natura, si rileva dall' osservazione

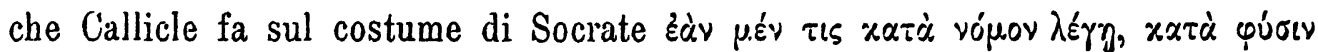

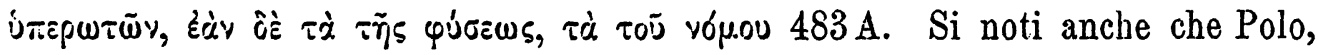
a cui si collega Callicle, è detto appunto uno dei fautori della legge, a cui

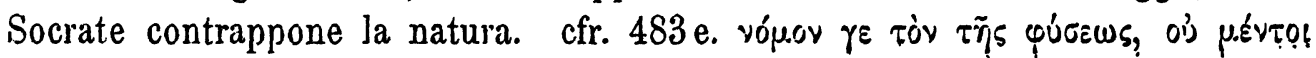

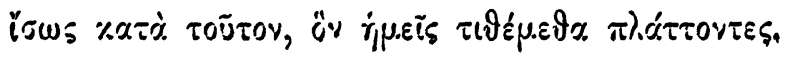


l'uomo entra in socictà, la cosa s'inverte"). La maggioranza conoscendo la propria debolezzia e fatta accorta che la sua salvezza sia nel porro leggi che sien freno alla prepotenza, le rinvigorisce colla sanzione di lode e di biasimo che associa all' osservanza e alla violazione di quelle: (osservazione che fa pensare alla modorna dottrina associazionistica inglese che nell' obbligatorietà della leggo morale vede un residuo dell' efficacia della sanzione sociale $\left.{ }^{8 *}\right)$. Così avviene che la così detta giustizia sociale disciplina il più forte e lo mansuefaccia come un giovane leone: sistema fittizio, artificioso, inventato dai più per la loro difesa, contrario alla natura, e che dà al più forte il diritto a ribellarsi contro questa impotenza dei più (ìcosvía-àvavôpía Gorg. 483 b.). Ponete che si dia l'opportunità, e codesto favorito da natura, fatto consapevole della sua forza, la romperà colle leggi e colle opinioni, inutili impedimenti, e riconquisterà, come un suo diritto, la gloriosa posizione che la natura gli aveva assegnata ${ }^{83}$ ). Il lioncello s'è scosso e ha fatto sentire la forza dei suo artigli.

Così il diritto di natura invocato da Ippia si converte per Callicle nella apoteosi del dispotismo e dell' assolutismo; nè a torto Polo d'Agrigento aveva, poco prima di lui, nello stesso dialogo, preso a difendere il tiranno Archelao di Macedonia. Callicle stesso ci presenta questo stato di natura come un irrompere di tutte le passioni, contro l'impotenza dei più (492 A ss.); tutto il resto è vana finzione di questi ${ }^{84}$ ). Data la distinzione del diritto naturale e del diritto positivo di Grozio, non è Rousseau ma Hobbes che ha ragione. E Calliclè è l'Hobbes dell' antichità.

Ma se così la scuola che propugnava il ritorno alla natura volgeva ad un intima dissoluzione, a non meno gravi e funeste conseguenze si giungeva dell' altra parte da chi aveva posto sul

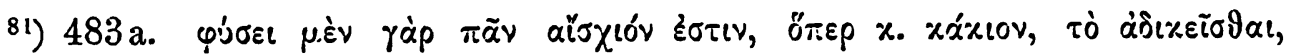

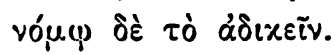

${ }^{82}$ ) Ib. b. cfr. Bain, Mental and Moral Science. London 1872, I, p. 394.

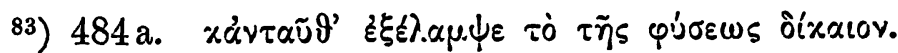

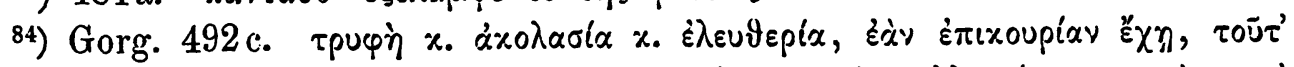

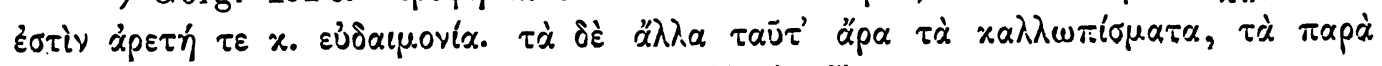

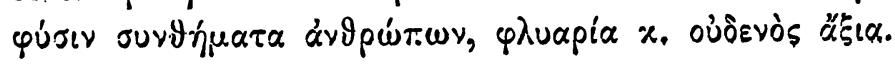


trono il Nomos re, come forza correttrice della selvaggia e rude natura. Come Callicle e Polo, sia pure anche Callicle, come crede il Welcker, un personaggio imaginario, rappresentano le tendenze dispotiche prevalenti nei circoli della gioventù aristocratica, dai quali escono Alcibiade e Crizia ${ }^{.85}$ ), Trasimaco e Glaucone formulano invece le conseguenze anarchiche che scendevano dalle teorie democratiche di Protagora e della sua scuola. La legge o la giustizia sociale, chi la guardi bene addentro, non è altro che ciò che giova al più forte ${ }^{86}$ ), 0 allo stato di qualunque forma esso sia. Poichè alla conclusione di Callicle si poteva obiettare ${ }^{87}$ ) che se le leggi limitano i più forti, impediscono anche l'arbitrio e la violenza; e poichè questa possibilità d'impedire richiede la maggioranza degli uomini, che per natura è più forte, così si potrebbe anche dire che è giusto per natura che non si dia arbitrio, o che la giustizia è posta da natura.

Ora questa difficolta è ripresa dailla dottrina di Trasimaco e di Glaucone che muove del fatto stesso delle legge e del diritto sociale, che altro non è se non l'utile della forza dominante. L'autorità della legge sociale è doppiamente illusoria; primo, perchè è una convenzione fatta da coloro che di per sè non hanno potenza di fare altrui male ${ }^{88}$ ) cioè la maggioranza dei deboli. Poi, perchè non è soltanto, secondo il dato di natura, come crede Callicle, ma anche per il diritto sociale che il fare. è migliore o più utile che il soffrire ingiustizia ${ }^{89}$ ); la quale è di fatto la condizione per-

s5) Welcker, Prodikos von Keos, Klein. Schr. II. p. 454. Che Callicle e Polo rappresentino dottrine diffuse nei circoli aristocratici di Atene si desume anche da quello the si dice nel Gorgia 482 E, $492 \mathrm{~d}$. cfr. Grote, Hist. of Gr. VI, 86.

${ }^{86)}$ Rep. I, 338c, 341 A, 343 b, che corrisponde alla dottrina che professò certo Trasimaco sulla giustizia umana. Hermias in Phaedr. p. 192 Ast. (cfr.

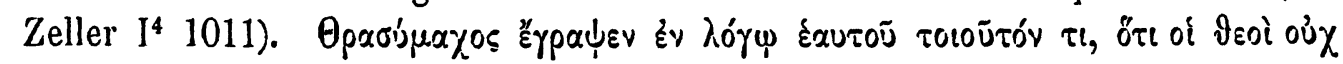

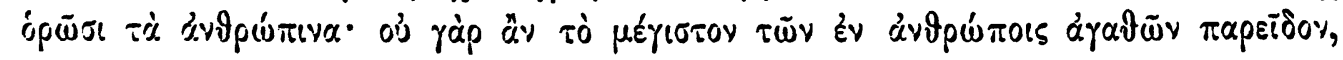

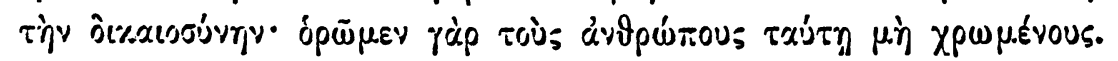

$\left.{ }^{87}\right)$ Koestlin, Gesch. d. Eth. I, p. 232 s.

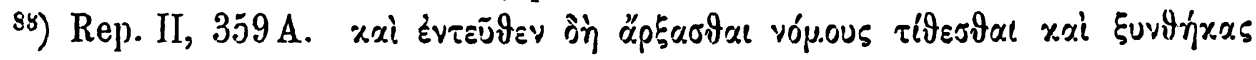

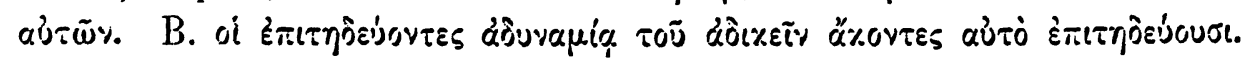

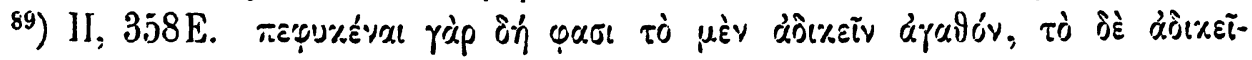
ร) $\%$ \% 
manente della vita socialo, dove la norma o la legge è l'utile del più forte, o della maggioranza dei deboli ${ }^{00}$ ). Il discorso di Trasimaco nel I o quello di Glaucone nel II della Repubblica, che è il compimento del primo, sebbene in apparenza affini a quello di Callicle, muovono dunque da opposte premesse. Non è un ritorno ad uno stato selvaggio di natura dove la prepotenza impera, ma la stessa giustizia sociale è la prova della prevalenza del forte sui deboli. Difatti per Trasimaco $i$ governanti mirano al bene proprio non a quello dei sudditi, come fanno $i$ pastori rispetto al loro gregge (343 B ss.). La così detta giustizia sociale è tutta a vantaggio dei più forti e a danno dei sottoposti, ed è solo apparente, poichè il giustó ci perde sempre. In questo senso la tirannide è la vera espressione della ingiustizia sociale.

Ma che la dottrina di Trasimaco non sia nella stessa direzione di quella di Callicle, si vede chiaro del commento che ne fa Glaucone. Questi riprende il discorso di Trasimaco su tre punti che riproducono, come pare, una dottrina allora comune e diffusa. 1 - Che cosa sia secondo i più la giustizia, e donde provenga (genesi del diritto sociale dalla maggioranza dei deboli confederati) - $2-$ Quelli che mantengono la giustizia (l'ordine sociale costituito), la mantengono per necessità non perchè sia un bene. - 3-A ragione pensano così perchè la vita dell' ingiusto è realmente più felice di quella del giusto ${ }^{91}$ ). Ora che codeste fossero teorie nascenti nella democrazia, lo dimostra la stessa dichiarazione che fa Glaucone, di essere assordato da mille altri oltre Trasimaco ${ }^{92}$ ); ed è facile il vedere come quest' ultima teoria differisca da quella di Callicle ${ }^{93}$ ) Non occorre risalire, con aspirazione antisociale, a un presunto stato di natura. Basta prendere la società come si trova per riconoscere nell' autorità venerata delle legge, mal dissimulati i tratti

90) I, 343 D - 344b. Per questo Glaucone dice che l'estremo della ingiustizia è nel passare per giusto senz'essere II, $361 \mathrm{~A}$; il che è appunto la condizione della vita sociale.

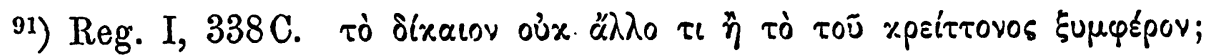

92) Ib. II, $358 \mathrm{C}$.

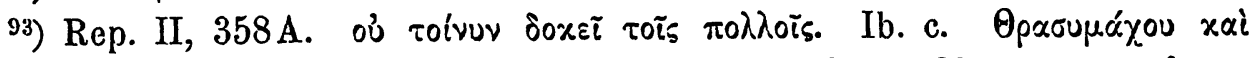

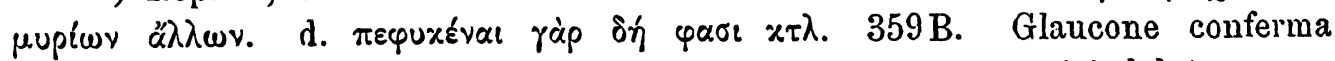
dunque che la teoria di Trasimaco è una teoria dei democratici del tempo. 
della violenza, da chiunque essa muova. E mentre la teoria di Callicle segna la rovina della scuola dello stato di natura, quella di Trasimaco abbatte la teoria del nomos. Callicle riesce al dispotismo assoluto, Trasimaco all' assoluta anarchia ${ }^{94}$ ). Ma poichè nello stato di anarchia l'individuo sciolto da ogni freno di costume e di legge, irrompe colla sua incomposta violenza, così l'una e l'altra dottrina riuscivano per opposte vie allo stesso resultato. Non vi è legge nè di natura nè sociale che valga: quello che a ciascuno par bene e giova questo è il giusto e il diritto, quando abbia la forza di farlo valere. Misura e giudice della vita morale non è nè la natura, nè la legge, ma l'individuo.

Questo spirito negativo s'estenderà naturalmente ad ogni ordine di tradizioni e di credenze. Se Protagora, con cauto riserbo, aveva sospeso ogni giudizio intorno all' esistenza degli Dei ${ }^{95}$ ), altri affermeranno arditamente che la religione è frutto d'un espediente politico e opera dei legislatori ${ }^{96}$ ) fin chè si giungerà facilmente ad ogni negazione con Diagora di Melos ${ }^{97}$ ). E già nella stessa letteratura vediamo i segni di codesto spirito audace, sopratutto in Euripide il poeta della giovine Atene ${ }^{98}$ ). Ma quello che più monta si è che codeste dottrine eran gia uscite dall' angusto cerchio delle scuole e si eran fatto largo nella vita pubblica, ed eran divenuti principi e massime politiche. Codesta diffusione, a torto negata dal Grote $^{99}$ ) ci è attestata da Tucidide. Questi che ci fa un così triste quadro delle condizioni morali della vita pubblica greca all'età della guerra del Peloponneso (III 82-83),

94) Köstlin op. cit. l. c.

${ }^{95}$ D. L. IX, 51. Theaet. $162 \mathrm{~d}$. Krische, Forschungen auf demGebiete der alten Phil. p. 132 ss.

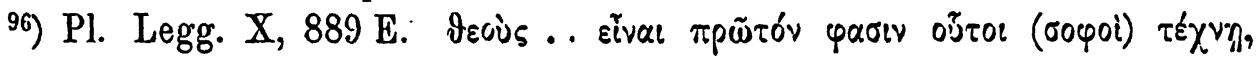

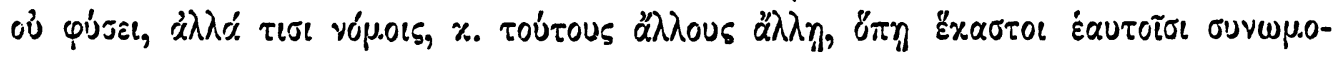

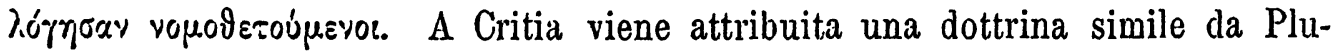
tarco (De Superst. 13) e da Sest. Empirico come contenuta in una tragedia „Sisifo", da altri attribuita ad Euripide (Zeller I, 1011).

${ }^{97}$ ) cfr. Zeller I ${ }^{4} 1011$ ss. Köstlin, Gesch. d. Eth. I, 226. Windelband, Gesch. d. alt. Phil. 72s.

98) Citiamo ad esempio Phoeniss. 503-23. Fragm. 19. Nauck.

99) Grote, History of Greece VI, 72 ss. 
e dí cosi sovero giudizio della vita morale in Atene dopo la pestilonza (II, 53), la quale, come avvenne in tutti $\mathrm{i}$ tempi, coruppe non mono gli animi che $i$ corpi, ci fa sentire come penetrasse sempre più nello spirito pubblico un modo di valutare gli atti lella vita privata e pubblica dal loro successo, e di pregiare solo il trionfo della forza. Cleone che nell' assemblea popolare sollecita gli Ateniesi a una inesorabile punizione, per lieve cagione, della città di Mitilene (III, 40); le aspre parole degli Ateniesi ai legati dell' Isola di Melos, senza ragione assalita da quelli, cioè che essi sapevano bene che solo dove le potenze contrastanti si bilanciano si suol seguire la norma della giustizia (óćxaıv); ma dove l'una prevalga, l'unica misura è l'utile (tò oupeșépov), e che come gli Dei giudicano per libera elezione, cosi gli uomini decidono secondo una necessità di natura, per la quale comanda il più forte ${ }^{100}$ ); e che infine essi, i Melii, quando si fossero levati in egual potenza, avrebbero potuto condursi egualmente, ci fanno sentire come fosse penetrato nella coscienza pubblica ed elevato a massima corrente nella vita politica il principio che la forza fa il diritto e legge è la prepotenza. Le teorie dei sofisti avevano-fatto cammino; nè basterà ad arrestarle l'opera riparatrice iniziale da Socrate, e continuata, contro gli ultimi sofisti, da Platone. La Grecia oramai s'avviava da lontano alla caduta della sua libertà nazionale; e da quella dinastia macedonica, di uno dei cui rè Callicle e Polo celebravano il potere dispotico, doveva sorgere fra breve colui che era destinato ad uccidere per sempre l'indipedenza della Grecia.

Lo Zeller ${ }^{101}$ ) ha giustamente rilevato che la questione se la

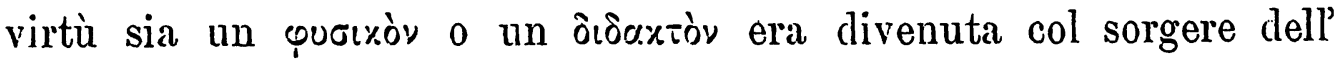
insegnamento dei Sofisti una questione favorita; poichè presso Senefonte (III 9, 1. IV , 2, 2) e nel principio del Menone platonico ${ }^{102}$ )

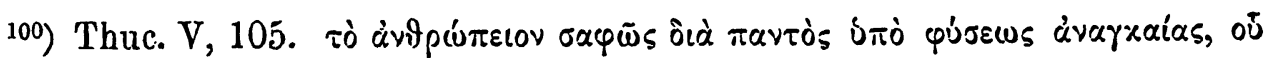

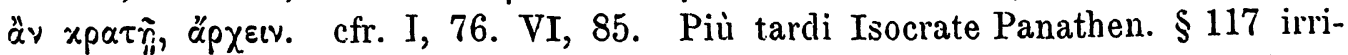
derà, alludendo a Platone, alla solitaria opinione di quei pochi che reputavano migliore il soffrire che il fare ingiustizia.

101) Zeller II ${ }^{4}$, I, 1889 p. 147.

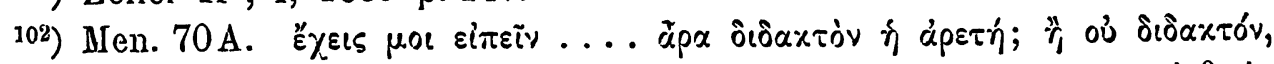

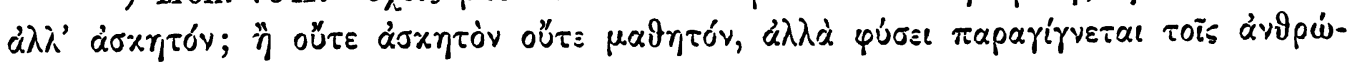

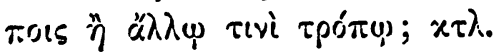


apparisce come tale. Solo deve aggiungersi che questa non è che una forma del problema più generale che occupa tutta la sofistica, cioè suoi rapporti morali della Natura e del Nomos nel suo senso più comprensivo. Ora mentre i Sofisti avevano accentuato il contrapposto fra il dato di natura e l'opera della vita civile, Socrate guarda il problema in questa forma particolare che più davvicino si collega coll' ufficio suo educativo. Difronte a questa duplice direzione del pensiero sofistico, Socrate prende una posizione speciale. Un pensatore che risolve la virtù nel sapere, e ne fa quindi un oggetto d'insegnamento, era logicamente condotto a ridurre al minimo l'importanza della natura nell' ordine morale. La insegnabilità della virtù, conseguenza dell' essere essa un sapere, ha poi anche la sua ragione nell' essere essa universalmente discibile. Ora questo suppone che, come le virtù in quanto scienze ne fanno una sola, così la differenza delle persone, delle età, dei sessi, non abbia alcuna efficacia nell' opera morale, e che tutto in questa debba ripetersi dalla educazione e dalla istruzione. Perciò Socrate a quella scuola dei Sofisti che riconosce la differenza naturale fra le virtù secondo l'età, il sesso, e le condizioni sociali ${ }^{103}$ ) contrappone la sua persuasione della unità delle virtù e della uguaglianza delle età e dei sessi rispetto ad essa ${ }^{104}$ ); dottrina nella quale è seguito da Antistene e degli altri socratici. La natura, per questo rispetto, non può nulla, e tutto deriva dalla istruzione e dalla educazione accessibile a tutti. Nello stesso senso combatte, presso Senofonte, l'opinione di coloro che, da Pindaro ad Ippia, tenerano a vile la cultura attribuendo ogni valore alle disposizioni naturali ${ }^{105}$ ), dimostrando ad essi che la natura, come fra gli animali cosi fra gli uomini,

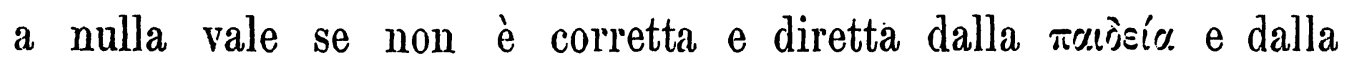
uáorro!s.

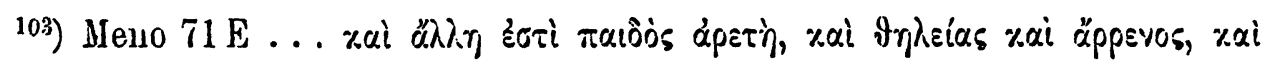

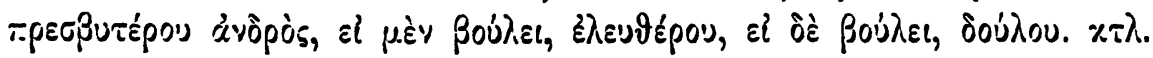

104) Xenoph. Symp. 2, 9. Pl. Men. 72 A ss. (Rep. V, 452E) Arist. Pol. I, 13. 1216 a. 20, cfr. Zeller $\mathrm{II}^{4}, 146$, e la mia dissertazione in Rivista di Filolog. classica. An. XII, fasc, 4-6, 1882.

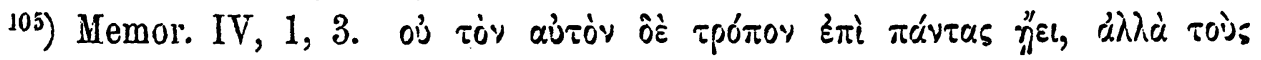

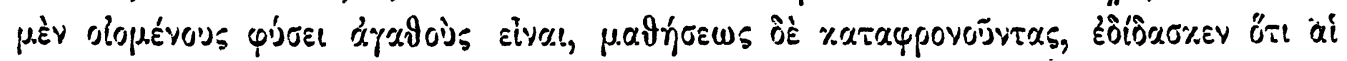

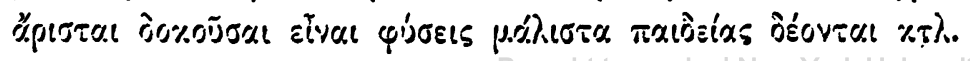


Ma dall' altro lato, il concetto che la virtì è scieuza e che come tale i discibile non conduce Socrate ad annullare il valore morale della natura. Senolonte dopo aver detto in qual modo Socrate si opponeva ai fautori esclusivi della natura, ci espone in qual modo rispondesse ai fautori esclusivi dell' educazione e della $\pi$ riduravice $\left.{ }^{106}\right)$ : Socrate ammette bensi che il giusto possa essere oggetto d'insegnamento ${ }^{107}$ ), e sul principio del colloquio, così ricco di pensiero, col giovine Eutidemo (Mem. IV. 2), sembra solo mettere in ridicolo l'autodidaxia di lui che qui piuttosto apparisce come un seguace delle abitudini e delle dottrine d'Ippia $\left.{ }^{108}\right)$ : il che a parer mio accenua e qualche confusione nel testo senofonte ${ }^{109}$ ). Senonche mentre a principio sembra che voglia dimostrare che l'arte politica,

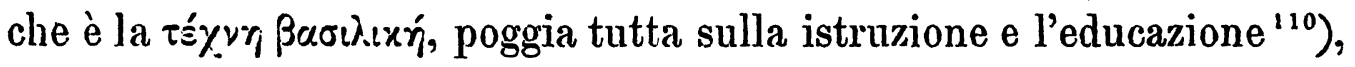
in ultimo fa sentire ad Eutidemo che quella stessa arte non è possibile esercitarla senza un dato naturale di giustizia ${ }^{11}$ ) che vale assai pì̀ del conoscere e del sapere.

Questa critica dei due concetti esclusivi dell' origine e del fondamento della virtù apre a Socrate la via a conciliarli in una dottrina più comprensiva che rispetti ad un tempo i diritti della natura e delle legge civile e dell' educazione. Se in tutti ci è una comune disposizione naturale alla virtù, quello che preme è lo svolgerla

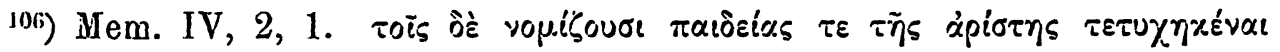

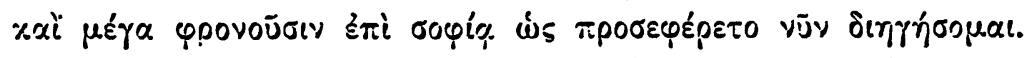

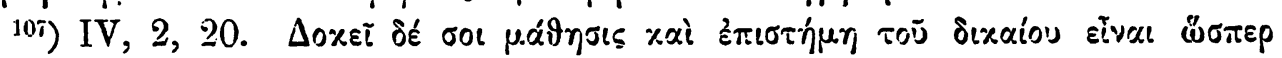

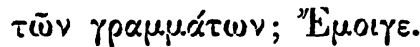

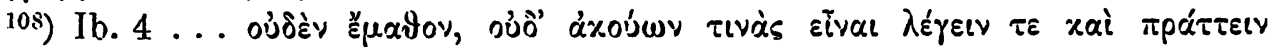

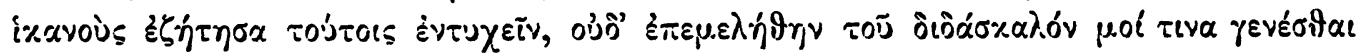

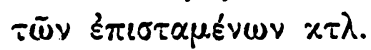

109) Perchè non so come Socrate potrebbe considerare come autodidatto

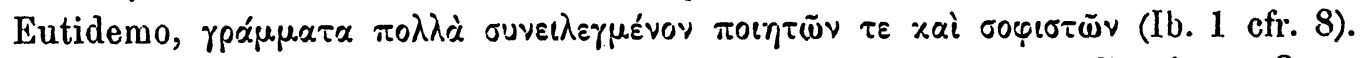

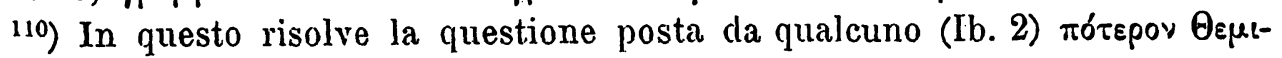

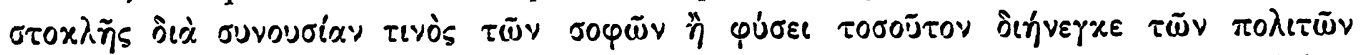

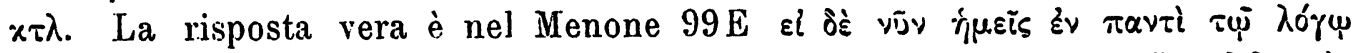

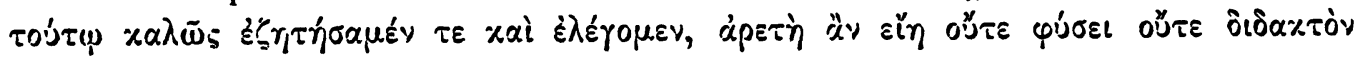

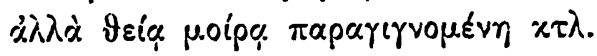

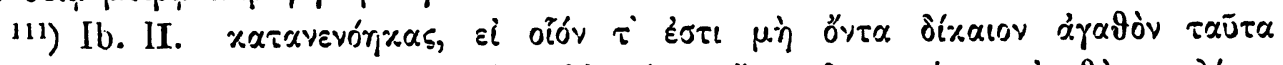

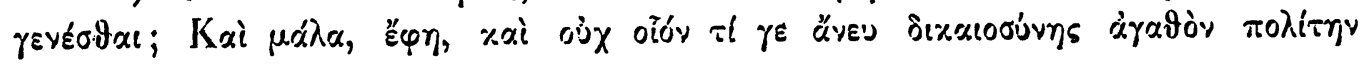
 
per via dell' esercizio e della educazione; la quale necessaria a tutti, è tanto più indispensabile se vi è una buona disposizione di natura $\left.{ }^{112}\right)$. E inversamente nessuna istituzione è efficace, se il seme non cada sopra adatto terreno ${ }^{113}$ ). Ogni virtù poggia dunque sulla yúocs ed anzi ha tanto valore che contro di essa non hanno valore talora il vóuos e l' Ěvos ${ }^{114}$ ), ma si accresce mediante l'esercizio e la cultura, o quel complesso di fattori che Socrate comprende nella $\pi$ rosía cioè volere e sapere a un tempo ${ }^{115}$ ). E tutta l'opera e la missione di Socrate è una conferma di fatto di questa sua fede nella natura e nella efficacia dell' insegnamento.

Alla stessa conciliazione fra la ẹúcs e il Nóuos Socrate del resto - era giunto per un altra via. D'accordo col Protagora del Teeteto platonico egli, contro ¡Ippia, afferma che il giusto è una cosa stessa col vóucuov ${ }^{116}$ ); identifica ciò̀ la giustizia colla osservanza della legge positiva, fondamento non solo della vita publica, ma ancora della privata (Mem. IV 4,17). Nè vale che gli stati mutino bene stesso le leggi, come gli obietta Ippia presso Senofonte (Ib. 14), poichè, non altrimenti che Protagor'a, Socrate gli risponde che finchè la legge è legge, l'osservarla è cosa buona e giusta. Se non che d'altra parte Socrate riconosce con Ippia la distinzione e il contrasto fra leggi positive e la legge naturale immutabile e universale. Ora questa doppia qualità delle leggi non scritte riconosciuta da Ippia e da Socrate le rivela d'origine divina; nella quale origine Socrate appunto vuol trovare il punto di conciliazione superiore fra le due opposte scuole della legge positive e della

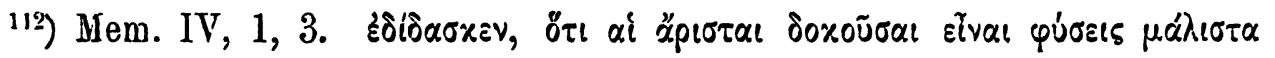

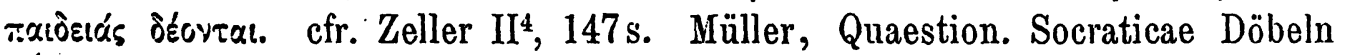
1877 p. XIXs.

113) I, 213 ss., IV, 2, 40. Plat. Theaet. $150 \mathrm{E}$, s.

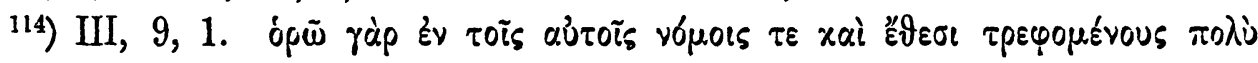

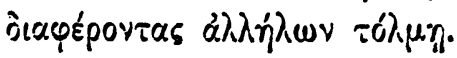

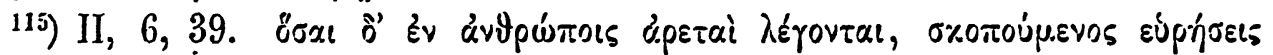

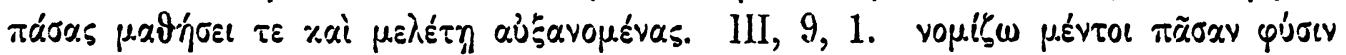

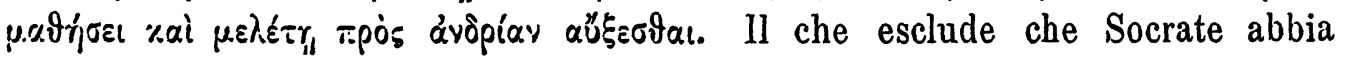

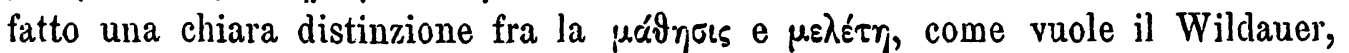
Psychol. d. Willens bei Sokr. 1877, I, 93. cfr. Zeller II', 148s.

$\left.{ }^{116}\right)$. cfr. Zeller II $^{4}, 150$. Ziegler, Gesch. d. Eth. I, 277. 
natura. Anche la natura is una legge, cioc legge divina; $e$ in questo senso veramente la giustizia si risolve in una osservanza dolle leggi" $\left.{ }^{17}\right)$. Ma questa stessa sanzione divina presuppone alla sua volta un dato naturale, e che la giustizia sia in un senso universale già prima che non sia la legge ${ }^{18}$ ). E per tal modo le opposte tesi d'Ippia e di Protagora si risolvono in una superiore armonia. Mentre la Sofistica dissolveva la coscienza morale; attaccando o la legge e la tradizione in nome della natura, 0 il fonlamento naturale in nome di un relativismo antropologico e sociale, Socrate mira a ricollocare la legge sul fondamento della natura. Il che gli è possibile partendo dal suo principio che lavirtì è scienza. Anche i Sofisti professavano che la virtù possa insegnarsi; ma il loro era un insegnamente empirico e pratico, e la virtù quindi un potere affatto relativo e variabile ${ }^{119}$ ). Per Socrate all' incontro è una potenza morale che ha bensì un fondamento in natura, ma un fondamento non variabile da individuo a individuo, da tempo a tempo, ma comune a tutti; e questo dato di natura deve poi essere svolto dalla educazione e dalla scienza diretta dai principii. In tal modo il pensiero Socratico mirava a soddisfare ad un tempo al bisogno sentito da alcuni di formare un diritto naturale contro il diritto costituito e sanzionato dallo Stato, e all' altra opposta esigenza dei novatori ribelli ad ogni principio assoluto di natura e fautori dell' individualismo politico.

L'opera conciliativa di Socrate non sembra sia stata continuata dalle scuole che lo seguirono e si svolsero da lui. Chi volesse seguire lo sviluppo di questi concetti nelle scuole successive a Socrate, vedrebbe come le cosi detto scuole Socratiche, specialmente Ciniche e Cirenaiche, segnino un ritorno alla idea della natura come criterio e norma della vita morale, e un alienarsi dalla vita sociale e dalla cultura. Abbiamo veduto come i Cinici continuino

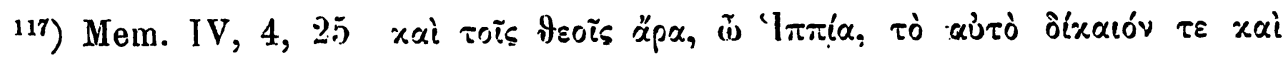

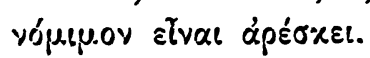

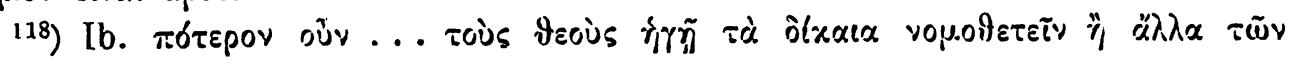

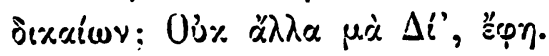

$\left.{ }^{119}\right)$ cfi. Siebeck, Ueber Sokr. Verhältn. z. Sophistik, in Untersuch. z. Philos. d. Griech. 2. Aufl. 1888 p. 33 ss. 
il Naturalismo etico di Prodico e d'Ippia, e sappiamo di Antistene che nel suo puocxós contrapponeva un monoteismo ilozoistico al Politeismo popolare prodotto dall' opinione e dalla convenzione ${ }^{120}$ ).

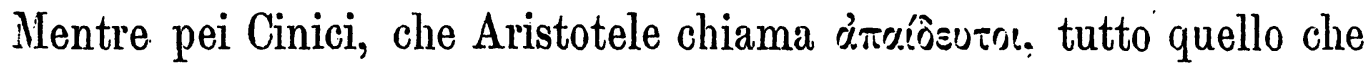
nasce dei rapporti civili è un joècrócopov e solo quello che è da natura vale moralmente, così del pari Aristippo, che si vanta di non avere patria alcuna ${ }^{121}$ ), si rivela, anche per questo rispetto, molto più un Sofista che un Socratico, ritornando alla antitesi inconciliabile fra la natura e la legge nell' ordine morale ${ }^{122}$ ). La sentenza attribuita ad Aristippo suona ben altra che presso Archelao, nè significa un ritorno ai principi della natura nel senso di Ippia; bensì rivela lo spirito negativo e dissolvente degli ultimi sofisti, in quanto nega l'esistenza d'ogni diritto naturale e positivo, e nel senso di Callicle e di Trasimaco nega il valore d'ogni legge sociale. Il che d'altronde era una conseguenza necessaria dell' Edonismo cirenaico, che è la più intera applicazione etica del principio individualistico della

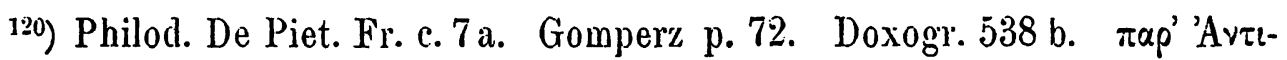

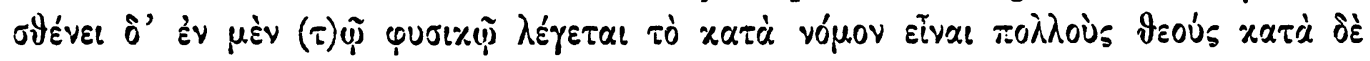

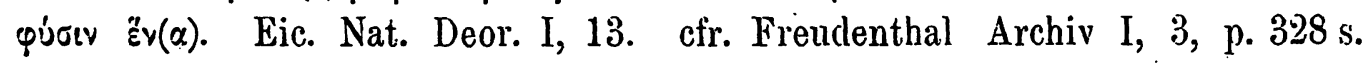
Dümmler Akademika p. 151.

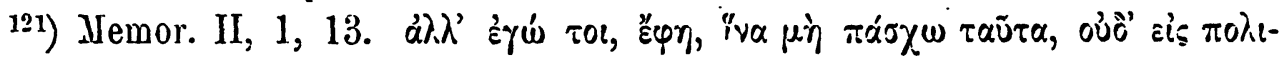

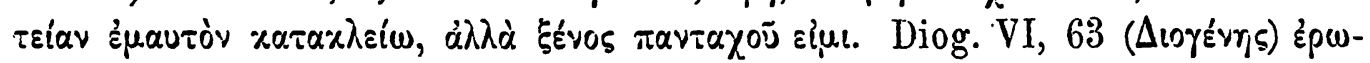

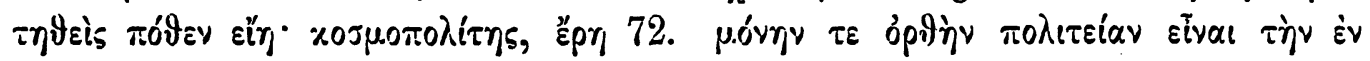

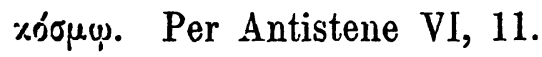

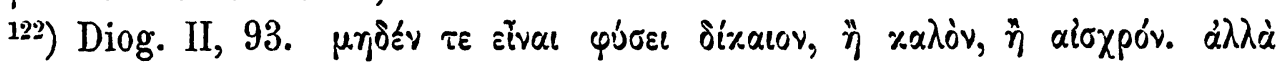

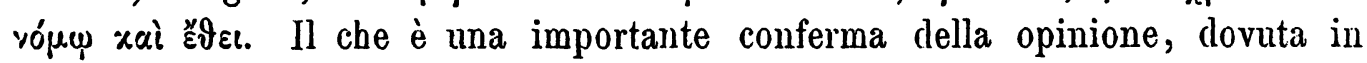
parte allo Schleiermacher, Gesch. d. Philos., 87. e più esplicitamente affermata dal Susemihl, N. Jahrb. f. Phil. 1882 p. 746, dallo Ziegler, Gesch. d. Eth., I, 14 s. Philos. Monatsh. 1888 p. $453 \mathrm{~s} .$, e in parte anche del Windelband, Gesch. der alt. Phil. 1888, 86 ss., che Aristippo e la scuola Cirenaica sieno piuttosto una continuazione della Sofistica e ad essa si colleghino, anzichè una derivazione de Socrate, come ammettono quasi tutti gli storici anche recenti, come il Köstlin, Gesch. d. Eth. I, 1887 p. 307 ss. Stein, Erkenntnissth. d. Stoa. 1888 p. 67 ss., e ora è sostenuto anche dello Zeller II $^{4}$ p. 369-75 (cfr. Müller, Quaestion. Socraticae 1877 p. XXV ss.). Poichè se è significativo che già

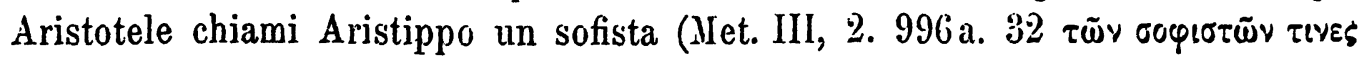

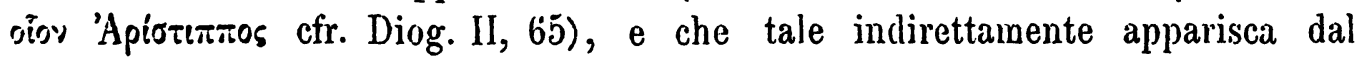
modo con cui Senofonte lo presenta a colloquio con Socrate (Nem. II, 1, 1), ̀̀ anche più eloquente il trovare attribuite a lui dottrine cosi caratteristiche dei Sofisti, come questa antitesi di moralità naturale, e moralità positiva. 
proposizione di Protagora; poichè il vero dato di natura non è il desiderio del bene, ma il desiderio del piacere (Diog. II, 88).

In un senso che si collega all' individualismo cosmopolitico dei Cinici e dei Cirenaici questo ritorno alla idea della natura come regola della vita morale riapparisce nelle scuole postaristoteliche,

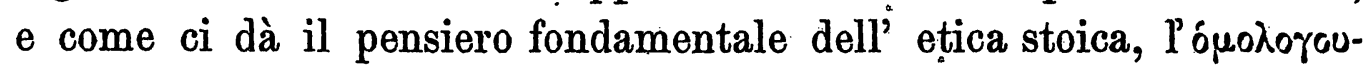

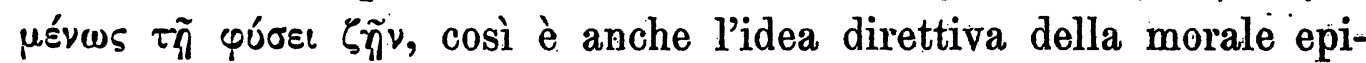
curea, e nell' una e nell' altra scuola s'accompagna naturalmente collo spirito di repugnanza ad una participazione alla vita publica, e con una tendenza ad esse comune, e assai più accentuata che non nei Cinici e in Aristippo, verso una morale cosmopolitica. Il concetto della cúós riapparisce più chiaro alla coscienza morale, quando la vita politica greca volge al suo tramonto. 